\title{
KINETIC APPROACH TO LONG TIME BEHAVIOR OF LINEARIZED FAST DIFFUSION EQUATIONS
}

\author{
MARíA J. CÁCERES * AND GIUSEPPE TOSCANI †
}

\begin{abstract}
We show that the rate of convergence towards the self-similar solution of certain linearized versions of the fast diffusion equation can be related to the number of moments of the initial datum that are equal to the moments of the self-similar solution at a fixed time. As a consequence, we find an improved rate of convergence to self-similarity in terms of a Fourier based distance between two solutions. The results are based on the asymptotic equivalence of a collisional kinetic model of Boltzmann type with a linear Fokker-Planck equation with nonconstant coefficients, and make use of methods first applied to the reckoning of the rate of convergence towards equilibrium for the spatially homogeneous Boltzmann equation for Maxwell molecules.
\end{abstract}

\section{Introduction}

This paper concerns some aspects of the rate of convergence to equilibrium for solutions to the Cauchy problem of the fast diffusion equation posed on the whole space $\mathbb{R}^{N}$

$$
\begin{gathered}
\frac{\partial v}{\partial \tau}=\Delta v^{m}, \quad y \in \mathbb{R}^{N}, \tau>0, \\
v(y, 0)=v_{0}(y),
\end{gathered}
$$

where $\frac{N}{N+2}<m<1$.

The long time asymptotics for equation (1.1) is described by the family of self similar source type Barenblatt-Pattle solutions

$$
B_{C}(y, \tau)=\tau^{-\frac{1}{m+1}}\left(C+\frac{1-m}{2 m}|y|^{2} \tau^{-\frac{2}{m+1}}\right)^{\frac{1}{1-m}},
$$

where the constant $C$ must be chosen in order to match the initial mass. Recent results on the subject allow to assert that the rate of convergence is sensitive to the choice of the initial datum, and it is believed that the values of its moments play an important role.

As a matter of fact, in the linear case described by the heat equation the rate of convergence towards the fundamental solution is improved by fixing the center of mass of the initial data. Moreover, one can fix a certain number of initial moments in order to recover a higher order asymptotic approximation for the solutions with a faster rate of convergence [18] [20]. In the same linear case it has been shown that mass-centering also speeds up the entropy decay [14]. These results suggest the possibility of detecting a more accurate asymptotic description even in the nonlinear case by fixing suitably a certain number of initial moments [34].

This phenomenon was first established by J.L. Vazquez in [31] for porous medium equations. He was able to prove that, while the support of a general solution takes the shape of the support of its corresponding shifted Barenblatt profile for large times, a faster convergence rate in $L^{\infty}$ towards such profile could be obtained in case of

\footnotetext{
*Departamento de Matemática Aplicada, Universidad de Granada, 18071 Granada, Spain. caceresg@ugr.es.

$\dagger$ Department of Mathematics, University of Pavia, via Ferrata 1, 27100 Pavia, Italy. giuseppe.toscani@unipv.it.
} 
solutions with radial symmetry. This convergence rate result is valid for the fast diffusion range as well.

In the fast diffusion case, the spatial translation invariance is related to an explicit eigenvalue of the linearization of the scaled equation in self-similar variables [12] [15]. Therefore, one expects an improvement of the rate of convergence for the fast diffusion equation by mass-centering to the rate $t^{-1}$ in $L^{1}$ in the translation dominated range, which is given by the next eigenvalue of the linearization. A nearly optimal convergence rate in the fast diffusion range, based on mass-centering, has been recently announced in [21] [24].

For the porous medium case, mass-centering has been recently used in [7] to show that the Euclidean Wasserstein distance between two compactly supported solutions of the one-dimensional porous medium equation having the same center of mass decays to zero for large times with a computable rate. As a consequence, mass-centering allows to detect an improved rate of convergence of solutions of the one-dimensional porous medium equation towards well centered self-similar Barenblatt profiles.

Let us denote the total mass of $v_{0}$ by

$$
M_{0}=\int_{\mathbb{R}^{N}} v_{0}(y) d y>0
$$

and its center of mass by

$$
M_{1}=\frac{1}{M_{0}} \int_{\mathbb{R}^{N}} y v_{0}(y) d y .
$$

Both quantities $M_{0}$ and $M_{1}$ are invariant with respect to time (see [30]). Hence, mass-centering means that we choose the initial datum with the same mass and first moment of the Barenblatt solution. Except in the linear case, however, higher order moments of the solution do not follow the same law of evolution of the higher order moments of a shifted in time Barenblatt profile, even if the same are equal at time $\tau=0$. As far as the second moment is concerned, a recent result establishes however an asymptotic equivalence between them [28].

Taking into account this asymptotic equivalence, it can be reasonably conjectured that information on the rate of decay in terms of moments can be obtained by considering linear or linearized versions of equation (1.1). Among others, we shall consider in the sequel a linear version of the fast diffusion equation (1.1),

$$
\begin{gathered}
\frac{\partial v(y, \tau)}{\partial \tau}=\operatorname{div}\left(m B_{C}(y, \tau)^{m-1} \nabla v(y, \tau)\right), \quad y \in \mathbb{R}^{N}, \tau>0 \\
v(y, 0)=v_{0}(y)
\end{gathered}
$$

where $B_{C}(y, \tau)$ is a Barenblatt solution suitably shifted in time. The rate of convergence to equilibrium for equation (1.4) will be studied in terms of the number of moments initially equal to thats of the Barenblatt solution.

Using the spatial-temporal scaling given in [11] [17], the initial value problem (1.4) can be rewritten as the initial value problem for the Fokker-Planck equation

$$
\begin{gathered}
\frac{\partial f}{\partial t}=\operatorname{div}\left[x f+m B_{C}(x)^{m-1} \nabla f\right] \quad x \in \mathbb{R}^{N}, t>0 \\
f(x, t=0)=f_{0}(x) \geq 0 .
\end{gathered}
$$


The initial value for the Fokker-Planck equation coincides with the initial value for the fast diffusion equation $\left(f_{0}=v_{0}\right)$. In equation (1.6) $B_{C}(x)$ is the Barenblatt solution (1.3) evaluated at time $\tau=1$,

$$
B_{C}(x)=\left(C+\frac{1-m}{2 m}|x|^{2}\right)^{-\frac{1}{1-m}} .
$$

Direct computations show that, for any fixed mass, the Fokker-Planck equation (1.6) has a unique stationary state given by (1.8).

A different linearization was first considered in [9]. There, the large-time asymptotic of linearized very fast diffusion equations with and without potential confinements were studied, by reckoning estimates for the spectral gap and drawing conclusions on the time decay of the solution. The results in [9] hold for arbitrary algebraically large diffusion speeds, provided the solutions have the mass-conservation property. Hence, the problem we will be dealing with, was not considered.

Likewise, this linearized fast diffusion equation was deeply investigated in [15] by Denzler and McCann, who were able to analyze its spectrum extracting sharp rates of asymptotic convergence to the Barenblatt profile. Our result recovers this rate of convergence by different methods (see Theorem 4.2 for more details). We must point out, as explained in [15], that, although our kinetic analysis is rigorous, the passage to linearization is clearly formal, and allows only to conjecture about analogous behavior for the solution to the original nonlinear equation.

For any given Barenblatt profile, characterized by the exponent $m$, higher moments of the solution stay uniformly bounded in time up to a critical exponent linked to the value of $m$. In addition to mass and momentum conservation, due to linearity, the higher moments of the solutions to equation (1.6) evolve in time in a closed form in terms of the lower order moments. This imply that the moments corresponding to two different initial data which are equal initially, remain equal to any subsequent time. Moreover, since the Barenblatt profile is a steady state to equation (1.6), if the initial datum has moments up some natural number $n>2$ equal to thats of the Barenblatt function (1.8), these moments remain constant in time. This property allows to compute precise rates of convergence to the stationary state in terms of the number of moments of the initial datum which are initially equal to thats of the equilibrium solution. The convergence rates of the linearized equations will be here derived in terms of a Fourier based metric which has been proven very useful in the finding of rates of convergence towards equilibrium in kinetic theory of rarefied gases, both in the conservative case [19] and in nonconservative one [3], [25].

These Fourier-based metrics $d_{s}$, for any $s>0$, are defined as

$$
d_{s}(f, g)=\sup _{\xi \in \mathbb{R}^{N}} \frac{|\hat{f}(\xi)-\hat{g}(\xi)|}{|\xi|^{s}}
$$

for any pair of probability measures in $\mathcal{P}_{s}\left(\mathbb{R}^{N}\right)$, where $\mathcal{P}_{s}\left(\mathbb{R}^{N}\right)$ is the set of probability measures with bounded $s$-moment and as usual, $\hat{f}$ is the Fourier transform of the density $f(x)$,

$$
\hat{f}(\xi)=\int_{\mathbb{R}^{N}} f(x) \mathrm{e}^{-i x \cdot \xi} d x
$$

By simple Taylor expansion one shows that the distance is well-defined and finite for any pair of probability measures with equal moments up to order $[s]$, where $[s]$ 
denotes the integer part of $s$. Moreover, in case $s \geq 1$ be an integer, it suffices equality of moments up to order $s-1$ for being $d_{s}$ finite. In this way, an (initially) bounded distance in our framework means an initial datum with a certain number of moments equal to thats of its corresponding steady state. In fact, $d_{s}$ with $s \geq 2$ topology is equivalent to the weak-star topology for measures plus convergence of moments up to order $[s]$, and can be related to the Wasserstein distance between probability measures [29].

The study of the convergence in terms of the distance (1.9) can be obtained through the analysis of its evolution. To this aim, the first tentative relies in the direct study of the evolution in time of the Fourier based distance using the linearized equation in Fourier transform. But, in view of its definition, the study of this evolution is a overcomplicated matter, due to the presence of a variable diffusion coefficient. To overcome this problem, instead of working on the Fokker-Planck equation directly, we will introduce a nonlinear kinetic model of Maxwell type [5] for which the recovering of the rate of decay in terms of the Fourier based distance is immediate. Then, the result for the Fokker-Planck equation will follow by a well-established asymptotic analysis recently introduced for analogous nonconservative Boltzmann equation [25]. This asymptotic procedure is reminiscent of the so-called grazing collision asymptotic. In kinetic theory of rarefied gases this asymptotic procedure became popular after the studies by C. Villani [32] [33], who established a rigorous connection between the elastic Boltzmann equation [13] and the Landau equation [23]. This procedure, which corresponds to concentrate collisions on the grazing ones, namely collisions which leave velocities unaffected, allows to recover in the limit Fokker-Planck (or more generally Landau-Fokker-Planck) equations. Other applications to the onedimensional dissipative Boltzmann equation can be found in [27].

In the rest of the paper we will assume the initial datum of unit mass, zero center of mass and unit second moment

$$
\int_{\mathbb{R}^{N}} f_{0} d x=1, \quad \int_{\mathbb{R}^{N}} x f_{0} d x=0, \quad \int_{\mathbb{R}^{N}}|x|^{2} f_{0} d x=1 .
$$

Since the mass and momentum are conserved in time, the solution to equation (1.6), which is initially a probability density of zero mean, so remains at any subsequent time $t>0$.

Our main results deal with the rate of decay to zero of solutions to different linearizations of the fast diffusion equation (1.1) in terms of the $d_{2+\delta}$ distance, where $\delta=\delta(m)$ is given in terms of the exponent $m$ of the fast diffusion. If equation (1.6) is considered, the typical result in one dimension reads

THEOREM 1.1. Let $f(v, t)$ be the solution to equation (1.6), with initial datum $f_{0}(v)$. Suppose in addition that

$$
d_{2+\delta}\left(f_{0}, B_{C}\right) \leq M<+\infty, \quad \delta<(3 m-1) /(1-m) .
$$

Then, the solution $f(v, t)$ converges exponentially to $B_{C}(x)$ in $d_{2+\delta}-m e t r i c$, and

$$
d_{2+\delta}\left(f(t), B_{C}\right) \leq d_{2+\delta}\left(f_{0}, B_{C}\right) \exp \left\{\frac{2+\delta}{2}\left(\frac{1-m}{m} \delta-\frac{3 m-1}{m}\right) t\right\} .
$$

Moreover, provided $3 / 5<m<1$ the rate of convergence in (1.12) increases in the interval $0<\delta \leq(5 m-3) /(2-2 m)$.

A careful reading of Theorem 1.1 clarifies the role of moments in the rate of convergence to equilibrium for solutions of equation (1.6). In fact, by definition, the 
$d_{s}(f, g)$ distance is finite as soon as the moments of $f$ and $g$ are equal up to the entire part $[s]$ of $s$. Hence, while $\delta=0$ in Theorem 1.1 only implies that there is mass-centering of the initial data, $\delta_{0}=(5 m-3) /(2-2 m) \leq 1$ implies that if at least the second moments of the initial data are equal, convergence to equilibrium is faster that before. Note that $\delta_{0}$ increases as soon as $m$ increases, and the convergence result is better if we are close to the linear case of the heat equation.

2. Preliminary results The main object of our analysis will be the study of the large-time asymptotic of the solution to the Fokker-Planck equation

$$
\begin{gathered}
\frac{\partial f}{\partial t}=\operatorname{div}\left[x f+\nabla\left(\left(\alpha^{2}+\beta^{2}|x|^{2}\right) f(x)\right)\right], \quad\left(x \in \mathbb{R}^{N}, t>0\right), \\
f(x, t=0)=f_{0}(x) \geq 0 . \quad\left(x \in \mathbb{R}^{N}\right)
\end{gathered}
$$

In equation (2.1) the constants $\alpha$ and $\beta$ are such that

$$
\alpha^{2}+\beta^{2}=\frac{1}{N}
$$

This condition implies that, if the initial datum $f_{0}(x)$ satisfies conditions (1.10), so does the solution at any subsequent time $t \geq 0$. It can be easily verified that equation (2.1) has a steady state with overpopulated tails, given by

$$
B_{\infty}(x)=C\left(\alpha^{2}+\beta^{2}|x|^{2}\right)^{-1-\left(2 \beta^{2}\right)^{-1}} .
$$

The constant $C$ in (2.3) has to be chosen to fit unit mass. In the one-dimensional case, equation (2.1), with $\alpha^{2}=\beta^{2}=1 / 2$ has been recently considered in [25] as the grazing limit of the one-dimensional dissipative Boltzmann equation studied in [2].

2.1. Fokker-Planck equations as linearization of confined fast diffusion equations

The linear Fokker-Planck equation (2.1) is strictly connected to the linearization of the fast diffusion equation (1.1). Consider in fact equation (1.1), with initial datum (1.2). It is well-known that (1.1)-(1.2) can be transformed into the fast diffusion equation with harmonic convection

$$
\begin{gathered}
\frac{\partial u}{\partial t}=\operatorname{div}\left(x u+\nabla u^{m}\right), \quad\left(x \in \mathbb{R}^{N}, t>0\right), \\
u(x, t=0)=u_{0}(x) \geq 0, \quad\left(x \in \mathbb{R}^{N}\right) .
\end{gathered}
$$

The transformation follows by the spatial-temporal scaling

$$
\begin{gathered}
x=\frac{y}{R(\tau)}, R(\tau)=((2-N+N m) \tau+1)^{1 /(2-N+N m)} \\
t(\tau)=\frac{1}{2-N+N m} \ln ((2-N+N m) \tau+1) \\
v(y, \tau)=R(\tau)^{-N} u\left(\frac{y}{R(\tau)}, t(\tau)\right)
\end{gathered}
$$


Applying (2.6)-(2.8) the similarity solution of the diffusion equation (1.1), given by (1.3) yields a equilibrium solution $B_{C}(x)$ of the Fokker-Planck equation, given by (1.8).

It is a simple exercise to show that the scaling (2.6)-(2.7) with

$$
v(y, \tau)=R(\tau)^{-N} f\left(\frac{y}{R(\tau)}, t(\tau)\right)
$$

transforms the linear diffusion equation (1.4) into (1.6) and $v_{0}(x)=f_{0}(y)$.

The linear Fokker-Planck equation (1.6) is obtained from (2.4), but other possible linearizations can be done from (2.4) as we will show now.

The linear Fokker-Planck equation (1.6) is a linearization of (2.4).- We recall $(2.4)$

$$
\frac{\partial u}{\partial t}=\operatorname{div}\left(x u+\nabla u^{m}\right), \quad\left(x \in \mathbb{R}^{N}, t>0\right)
$$

and consider a state $u(x, t) \geq 0$ close to the equilibrium $B_{C}(x)$, i.e., $u-B_{C}=O(\epsilon)$ in an appropriate topology. Thus we approximate the nonlinear term as

$$
\nabla u^{m}=m u^{m-1} \nabla f \approx m B_{C}^{m-1} \nabla u .
$$

Identifying $u$ with $f$, we just obtain (1.6)

$$
\frac{\partial f}{\partial t}=\operatorname{div}\left[x f+m B_{C}(x)^{m-1} \nabla f\right] \quad x \in \mathbb{R}^{N}, t>0 .
$$

Since

$$
B_{C}(x)^{m-1}=C+\frac{1-m}{2 m}|x|^{2},
$$

one concludes with

$$
m \nabla\left[B_{C}(x)^{m-1} f\right]=m B_{C}(x)^{m-1} \nabla f+(1-m) x f .
$$

Hence (1.6) can be rewritten as

$$
\frac{\partial f}{\partial t}=\operatorname{div}\left[m x f+m \nabla\left(B_{C}(x)^{m-1} f\right)\right] .
$$

Other possible linearization of (2.4).- Let us set

$$
f(x, t)=\frac{1}{1+\epsilon}\left(B_{C}(x)+\epsilon \rho(x, t)\right),
$$

for $\epsilon>0$ small and " $\rho=O(1)$ ". Assuming mass-conservation

$$
\int_{\mathbb{R}^{N}} B_{C}(x) d x=\int_{\mathbb{R}^{N}} f(x, t) d x=M, t \geq 0,
$$

we have

$$
\int_{\mathbb{R}^{N}} \rho(x, t) d x=M, t \geq 0
$$


Inserting (2.12) into (2.4), computing the derivative with respect to the parameter $\epsilon$ and setting $\epsilon=0$ gives the linearization:

$$
\begin{gathered}
\frac{\partial \rho}{\partial t}=\operatorname{div}\left[x \rho+m \nabla\left(B_{C}(x)^{m-1} \rho\right)\right] \quad x \in \mathbb{R}^{N}, t>0, \\
\rho(x, t=0)=\rho_{0}(x) \geq 0 .
\end{gathered}
$$

with

$$
\int_{\mathbb{R}^{N}} \rho_{0} d x=M
$$

Note that both linearizations (2.14) and (2.11) have the same diffusion term, and differ only for the presence of the constant in front of the convection term. This difference reflects into the stationary solution, which coincides with the equilibrium Barenblatt solution (1.8) in equation (2.11), while for (2.14) is given by

$$
\rho_{\infty}(x)=D B_{C}^{2-m}(x),
$$

where the constant $D$ is fixed in terms of the initial mass. Due to their similar form, both equations (2.14) and (2.11) can be studied by a unified treatment.

Both linearizations of (2.4) can be written as the Fokker-Planck equation (2.1).By a simple scaling argument, one can finally show that both equations (2.14) and (2.11) fall into the form of equation (2.1). Hence we will focus in the large-time behavior of solutions to this last equation, and in consequence we obtain the longtime behavior of both linearizations of (2.1).

First of all, we consider equation (2.14). Using equality (2.10)

$$
m B_{C}(x)^{m-1}=m C+\frac{1-m}{2}|x|^{2} .
$$

Note that, provided $m>(N-2) / N$, the coefficient of $|x|^{2}$ is less than $1 / N$. If $\rho(x, t)$ solves (2.14), let us set $\rho(x, t)=\delta^{N} g(\delta x, t)$, where $\delta$ is a constant to be chosen. Then if $y=\delta x, g(y, t)$ solves

$$
\begin{gathered}
\frac{\partial g}{\partial t}=\operatorname{div}\left[y g+\nabla\left(\left(\delta^{2} m C+\frac{1-m}{2}|y|^{2}\right) g(y)\right)\right], \quad\left(y \in \mathbb{R}^{N}, t>0\right), \\
g(y, t=0)=\delta^{-N} f_{0}\left(\delta^{-1} y\right) \geq 0 \quad\left(y \in \mathbb{R}^{N}\right) .
\end{gathered}
$$

Therefore, $g(y, t)$ satisfies equation (2.1) for a convenient $\delta$ in such a way that $\alpha^{2}+\beta^{2}=$ $\frac{1}{N}$, where $\alpha^{2}=\delta^{2} m C$ and $\beta^{2}=\frac{1-m}{2}$. By a simple computation we show that $\delta$ has to be chosen in the following way

$$
0<\delta^{2}=\frac{1}{C m}\left(\frac{1}{N}-\frac{1-m}{2}\right)
$$

The same strategy applies to equation (2.11). The only difference is that here we have to scale only the time. Let us set $f(x, t)=h(x, m t)$. Then, if $\tau=m t, h(x, \tau)$ solves

$$
\frac{\partial h}{\partial \tau}=\operatorname{div}\left[x h+\nabla\left(\left(C+\frac{1-m}{2 m}|x|^{2}\right) h(x)\right)\right], \quad\left(x \in \mathbb{R}^{N}, \tau>0\right),
$$




$$
h(x, \tau=0)=f_{0}(x) \geq 0 . \quad\left(x \in \mathbb{R}^{N}\right)
$$

In this case,

$$
\alpha^{2}=\frac{1}{N}-\frac{1-m}{2 m} ; \quad \beta^{2}=\frac{1-m}{2 m}
$$

Note that the bound $\beta^{2}<1 / N$ implies $m>N /(N+2)$. By resorting to the definition of the Fourier based distance (1.9), any result on the large-time behavior of the distance between the solution to the Fokker-Planck equation (2.1) and its stationary state (2.3) can be easily translated into the corresponding result on the large-time behavior of the distance between the solution to the Fokker-Planck equation (2.14) or (2.11) and their stationary Barenblatt solutions. In fact, the relation $\rho(x, t)=\delta^{N} g(\delta x, t)$ implies $\hat{g}(\xi)=\hat{\rho}(\delta \xi)$, and

$$
d_{s}\left(g, B_{\infty}\right)=\sup _{\xi \in \mathbb{R}^{N}} \frac{\left|\hat{\rho}(\delta \xi)-\hat{B_{\infty}}(\delta \xi)\right|}{|\xi|^{s}}=\frac{\left|\hat{\rho}(\xi)-\hat{\rho_{\infty}}(\xi)\right|}{|\xi|^{s}} \delta^{s}
$$

where $\delta$ is given by (2.20). Analogous result holds for equation (2.11).

REMARK 2.1. The previous computation emphasize a noticeable difference between the linearized fast diffusion equation (2.14) and the linear one given by equation (2.11). In the former case, the condition $\beta^{2}<1 / N$ is satisfied if

$$
m>\frac{N-2}{N}
$$

while, in the latter case, the same condition on $\beta$ holds if

$$
m>\frac{N}{N+2} .
$$

Thus, finite mass for the Barenblatt solution is enough to study the linearized equation (2.14), while finite second moment for the Barenblatt profile is needed to study the linear equation (2.11).

\subsection{On moments of the Barenblatt solution}

The previous analysis shows that the Fokker-Planck equation (2.1) contains various linear or linearized versions of scaled fast diffusion equations. This fact depends clearly on the structure of the steady Barenblatt solution. For the sake of completeness, we shall recall in the following some of these properties. First, we recover the relationship between the zero order moment (mass) of the Barenblatt solution and its second one (the temperature) (see [28]). Let $u(x, t)$ be a solution to equation (2.4). For any given $r \in \mathbb{N} \cup\{0\}$, let us set

$$
M_{r}(t)=\int_{\mathbb{R}^{N}}|x|^{r} u(x, t) d x
$$

Direct computations show that

$$
\frac{d}{d t} M_{2}(t)=-2 M_{2}(t)+2 N \int_{\mathbb{R}^{N}} u^{m} d x,
$$

Since the Barenblatt is a steady solution to equation (2.27), its second moment $M_{2}$ satisfies

$$
M_{2}+2 N \int_{\mathbb{R}^{N}} B_{C}^{m} d x
$$


Using the expression of the Barenblatt solution (1.8) we find

$$
\int_{\mathbb{R}^{N}} B_{C}^{m} d x=\int_{\mathbb{R}^{N}}\left(C+\frac{1-m}{2 m}|x|^{2}\right) B_{C} d x=C M_{0}+\frac{1-m}{2 m} M_{2} .
$$

Hence

$$
M_{2}\left(\frac{N(1-m)}{m}-2\right)+2 N C M_{0}=0
$$

and therefore

$$
C M_{0}=\left(\frac{1}{N}-\frac{1-m}{2 m}\right) M_{2}
$$

If we fix the values of the moments, $M_{0}=M_{2}=1$, we then obtain for $C$ the value

$$
C=\frac{1}{N}-\frac{1-m}{2 m}
$$

found in (2.23).

REMARK 2.2. Since both $M_{0}$ and $M_{2}$ are nonnegative, formula (2.28) implies the restriction

$$
\frac{1}{N}-\frac{1-m}{2 m}>0
$$

Consequently the second moment is finite if and only if $m>\frac{N}{N+2}$.

The precise values of higher order (and bounded) moments of the Barenblatt solution can be evaluated recursively starting from the equality

$$
\frac{d}{d t} M_{n}(t)=-n M_{n}(t)+n(n-2+N) \int_{\mathbb{R}^{N}}|x|^{n-2} u^{m} d x
$$

where $2<n \in \mathbb{N}$. As before, we use the fact the Barenblatt is a steady solution to equation (2.29), which implies

$$
M_{n}=(n-2+N) \int_{\mathbb{R}^{N}} B_{C}^{m} d x
$$

We find

$$
\int_{\mathbb{R}^{N}}|x|^{n-2} B_{C}^{m} d x=\int_{\mathbb{R}^{N}}|x|^{n-2}\left(C+\frac{1-m}{2 m}|x|^{2}\right) B_{C} d x=C M_{n-2}+\frac{1-m}{2 m} M_{n} .
$$

Finally, using the expression of $C$ found in (2.23),

$$
\left(\frac{1}{n-2+N}-\frac{1-m}{2 m}\right) M_{n}=\left(\frac{1}{N}-\frac{1-m}{2 m}\right) M_{n-2} .
$$

This relation also gives the maximum moment of the Barenblatt solution which is bounded. In fact, the procedure can be iterated up to the value of $n$ for which the coefficient of $M_{n}$ is greater than zero. A further interesting property of Barenblatt solutions is related to the scaling of variables. We used this scaling in the previous section to normalize the constants $\alpha$ and $\beta$. We have 
LEMMA 2.1 (Relation between Barenblatt solutions). Let $B$ a Barenblatt solution with constant $C$ and mass and energy $M_{0}$ and $M_{2}$ respectively. Then, if $b^{m-1} a^{2}=1$, the function $\bar{B}(x)=b B(a x)$ is a Barenblatt solution with

$$
\bar{M}_{0}=\frac{b}{a^{N}} M_{0}, \bar{M}_{2}=\frac{b}{a^{N+2}} M_{2} \quad \text { and } \quad \bar{C}=b^{m-1} C .
$$

Proof. Expressions for mass and energy are direct computations. We will focus only in proving that $\bar{B}$ is really a Barenblatt solution. We have

$$
\bar{B}(x)^{m-1}=b^{m-1}\left(C+\frac{1-m}{2 m} a^{2}|x|^{2}\right)=b^{m-1} C+b^{m-1} \frac{1-m}{2 m} a^{2}|x|^{2} .
$$

Then, since $b^{m-1} a^{2}=1$ the previous equality can be written as

$$
\bar{B}(x)=\left(b^{m-1} C+\frac{1-m}{2 m}|x|^{2}\right)^{\frac{-1}{1-m}} .
$$

$\square$

\subsection{Remark on the heat equation}

In order to understand the role of the Fourier based distance in the reckoning of the large-time behavior of the linear Fokker-Planck equation (2.1) we recall briefly how this distance can be used in the case of the linear heat equation. The result that follows is contained in [20], where it appears as a simple example to justify application of the distance $d_{s}$ to the rate of convergence towards the Gaussian density in the central limit theorem. Another application of the distance (1.9) to the classical Fokker-Planck equation can be found in [10]. These results follow in consequence of the fact that in these cases there exists an explicit solution, which allows for exact computations. Due to its importance in applications to the linear Fokker-Planck equations, we briefly describe how this distance works.

It is well known that a solution of the heat equation

$$
\begin{gathered}
\frac{\partial u}{\partial t}=\Delta u, \quad x \in \mathbb{R}^{N}, \\
u(x, 0)=u_{0}(x),
\end{gathered}
$$

where $u_{0}(x)$ is a probability density function satisfying conditions (1.10), behaves asymptotically in time as the fundamental solution of $(2.31)$, which is given by the Gaussian density

$$
\omega_{2 t}(x)=\frac{1}{\sqrt{4 \pi t}} \exp \left\{-\frac{|x|^{2}}{4 t}\right\} .
$$

The rate of convergence, that in $L^{2}$ norm is governed by $t^{-N / 2}$, can be improved as soon as we have more information on the moments of the initial data. A simple proof of this result follows by using the Fourier based distance (1.9). More precisely, let $u_{1}$ and $u_{2}$ be solutions of $(2.31)$, corresponding to initial data $u_{0,1}$ and $u_{0,2}$ respectively, 
and let $s>0$ be such that $d_{s}\left(u_{0,1}, u_{0,2}\right)$ is finite. By Fourier transform, we get for $i=1,2$

$$
\partial_{t} \widehat{u_{i}}(t, \xi)=-|\xi|^{2} \widehat{u_{i}}(t, \xi) .
$$

Solving the ordinary differential equation leads to

$$
\left|\widehat{u_{1}}(t, \xi)-\widehat{u_{2}}(t, \xi)\right|^{2}=\exp \left(-2 t|\xi|^{2}\right)\left|\widehat{u_{0,1}}(\xi)-\widehat{u_{0,2}}(\xi)\right|^{2} .
$$

It follows that the $L^{2}$ norm is estimated by using the Parseval equality

$$
\begin{aligned}
\int_{\mathbb{R}^{N}}\left|u_{1}(t, x)-u_{2}(t, x)\right|^{2} d x & =(2 \pi)^{-N} \int_{\mathbb{R}^{N}}\left|\widehat{u_{1}}(t, \xi)-\widehat{u_{2}}(t, \xi)\right|^{2} d \xi \\
& =(2 \pi)^{-N} \int_{\mathbb{R}^{N}} \exp \left(-2 t|\xi|^{2}\right)\left|\widehat{u_{0,1}}(\xi)-\widehat{u_{0,2}}(\xi)\right|^{2} d \xi \\
& \leq d_{s}\left(u_{0,1}, u_{0,2}\right)^{2} F(t) .
\end{aligned}
$$

The function of time $F(t)$, that is equal to

$$
F(t)=(2 \pi)^{-N} \int_{\mathbb{R}^{N}} \exp \left(-2 t|\xi|^{2}\right)|\xi|^{2 s} d \xi
$$

is bounded and can be computed explicitly to give (2.34). In fact, if

$$
\begin{gathered}
C=(2 \pi)^{-N} \int_{\mathbb{R}^{N}} \exp \left(-2|\xi|^{2}\right)|\xi|^{2 s} d \xi, \\
F(t)=C t^{-(s+N / 2)} .
\end{gathered}
$$

Therefore, it follows that

$$
\left\|\left(u_{1}-u_{2}\right)(t, \cdot)\right\|_{L^{2}\left(\mathbb{R}^{N}\right)}^{2} \leq d_{s}\left(u_{0,1}, u_{0,2}\right)^{2} C t^{-(s+N / 2)},
$$

where the constant $C$ is explicitly computable [20].

In particular, as $s \rightarrow 0$, we recover from (2.34) the usual $t^{-N / 2}$ decay rate. The decay rate in (2.34) can be rephrased in terms of moments. Let $s=m+\delta$, where $m$ is an integer and $0 \leq \delta<1$. Since the Gaussian density (2.33) is a solution to the heat equation, looking at the definition of the metric $d_{s}$, if the initial datum $u_{0}(x)$ has the same moments of the fundamental solution $\omega_{2}(x)$ up to $p=m$, the $d_{s}$-distance $d_{s}\left(u_{0}, \omega_{2}\right)$ is bounded, and the rate of convergence becomes $t^{-(s+N / 2)}$.

As the previous example shows, one of the interesting features of the distance (1.9) is that it can be used through interpolation to obtain convergence in stronger spaces. This property has been discovered in [6], and subsequently used in various applications to the dissipative Boltzmann equation [3].

\subsection{A property of the Fourier-based distance}

Various properties of the distance (1.9) were collected in papers [19], [29], where the interested reader can achieve information on the relationship of this norm to other more familiar equivalent norms used both in probability theory and in mass transportation. For the purposes of this paper, however, further properties are needed. We prove the following 
LEMmA 2.2. Let $\left\{f_{n}(x)\right\}_{n \geq 0}$ and $\left\{g_{n}(x)\right\}_{n \geq 0}, x \in \mathbb{R}^{N}$, be two sequences satisfying conditions (1.10) and such that $f_{n} \rightarrow f, g_{n} \rightarrow g$. Suppose in addition that, for some $r>2$

$$
\int_{\mathbb{R}^{n}}|x|^{r} f_{n}(x)<+\infty, \quad \int_{\mathbb{R}^{n}}|x|^{r} g_{n}(x)<+\infty
$$

and, in addition

$$
d_{r}\left(f_{n}, g_{n}\right)<+\infty
$$

Then, for all $s<r$,

$$
d_{s}(f, g) \leq \liminf d_{s}\left(f_{n}, g_{n}\right)
$$

Proof. By definition, $d_{r}\left(f_{n}, g_{n}\right)$ is bounded if all moments of order less or equal than $[r]$ of $f_{n}$ and $g_{n}$ are equal. Since $f_{n} \rightarrow f$ and $g_{n} \rightarrow g$, condition (2.35) implies that, for any $s<r$

$$
\int_{\mathbb{R}^{n}}|x|^{s} f_{n}(x) \rightarrow \int_{\mathbb{R}^{n}}|x|^{s} f(x), \quad \int_{\mathbb{R}^{n}}|x|^{s} g_{n}(x) \rightarrow \int_{\mathbb{R}^{n}}|x|^{s} g(x) .
$$

Thus, $d_{r}(f, g)=D$ is bounded. Now, consider that

$$
\begin{gathered}
\sup _{\xi \in \mathbb{R}^{N}} \frac{|\widehat{f}(\xi)-\widehat{g}(\xi)|}{|\xi|^{s}} \leq \\
\inf _{n \geq k} \sup _{\xi \in \mathbb{R}^{N}} \frac{\left|\widehat{f}_{n}(\xi)-\widehat{g}_{n}(\xi)-(\widehat{f}(\xi)-\widehat{g}(\xi))\right|}{|\xi|^{s}}+\inf _{n \geq k} \sup _{\xi \in \mathbb{R}^{N}} \frac{\left|\widehat{f}_{n}(\xi)-\widehat{g}_{n}(\xi)\right|}{|\xi|^{s}} .
\end{gathered}
$$

Now, from the inequality

$$
\sup _{|\xi| \leq \delta} \frac{\left|\widehat{f}_{n}(\xi)-\widehat{g}_{n}(\xi)\right|}{|\xi|^{s}} \leq \sup _{|\xi| \leq \delta} \frac{\left|\widehat{f}_{n}(\xi)-\widehat{g}_{n}(\xi)\right|}{|\xi|^{r}}|\delta|^{r-s}
$$

for any given $\epsilon>0$ there exists $\delta>0$ such that, for all $n$

$$
\sup _{|\xi| \leq \delta} \frac{\left|\widehat{f}_{n}(\xi)-\widehat{g}_{n}(\xi)-(\widehat{f}(\xi)-\widehat{g}(\xi))\right|}{|\xi|^{s}} \leq(C+D)|\delta|^{r-s} \leq \epsilon .
$$

Likewise, there exists $R>0$ such that, for all $n$

$$
\sup _{|\xi| \geq R} \frac{\left|\widehat{f}_{n}(\xi)-\widehat{g}_{n}(\xi)-(\widehat{f}(\xi)-\widehat{g}(\xi))\right|}{|\xi|^{s}} \leq 4 / R^{s} \leq \epsilon .
$$

Thus, for any $\epsilon>0$ we can find $\delta$ and $R$ such that

$$
\sup _{\xi \in \mathbb{R}^{N}} \frac{\left|\widehat{f}_{n}(\xi)-\widehat{g}_{n}(\xi)-(\widehat{f}(\xi)-\widehat{g}(\xi))\right|}{|\xi|^{s}} \leq
$$




$$
\begin{gathered}
\max \left\{\epsilon \sup _{\delta \leq|\xi| \leq R} \frac{\left|\widehat{f}_{n}(\xi)-\widehat{g}_{n}(\xi)-(\widehat{f}(\xi)-\widehat{g}(\xi))\right|}{|\xi|^{s}}\right\} \leq \\
\max \left\{\epsilon, \sup _{\delta \leq|\xi| \leq R} \frac{1}{\delta^{s}}\left(\left|\widehat{f}_{n}(\xi)-\widehat{f}(\xi)\right|+\left|\widehat{g}_{n}(\xi)-\widehat{g}(\xi)\right|\right)\right\} .
\end{gathered}
$$

Now, since $f_{n} \rightarrow f$ and $g_{n} \rightarrow g$ we have

$$
\forall \xi, \widehat{f_{n}}(\xi) \longrightarrow \widehat{f}(\xi)
$$

and since $f_{n}$ satisfies conditions $(1.10),\left|D^{2} \widehat{f}_{n}(\xi)\right| \leq 1$ and $D \widehat{f}_{n}(0)=0$. Thus $\left\{\widehat{f}_{n}\right\}_{n \geq 0}$ is uniformly equi-continuous on the compact set $\{\delta \leq|\xi| \leq R\}$. By Ascoli's theorem, this entails that $\sup _{\delta \leq|\xi| \leq D}\left|\widehat{f}_{n}(\xi)-\widehat{f}(\xi)\right|$ goes to 0 . Same conclusion for the sequence $g_{n}$. Finally, there exists $n_{0} \geq 0$ such that for $n \geq n_{0}$,

$$
\max \left\{\epsilon, \sup _{\delta \leq|\xi| \leq R} \frac{1}{\delta^{s}}\left(\left|\widehat{f}_{n}(\xi)-\widehat{f}(\xi)\right|+\left|\widehat{g}_{n}(\xi)-\widehat{g}(\xi)\right|\right)\right\} \leq \epsilon
$$

This concludes the proof.

$\square$

\section{The one-dimensional Fokker-Planck equation}

\subsection{A nonconservative kinetic model}

This section concerns the introduction of some nonlinear kinetic model of Boltzmann type, which is related to the Fokker-Planck equation (2.1) in the so-called grazing collision limit procedure [32], [33]. Similar ideas were introduced in [25] in order to obtain information on the self-similar profile of one-dimensional nonlinear Boltzmann equations of Maxwell type, in the case of lack of conservation of the energy. The main advantage in working with a Boltzmann type equation relies in the possibility to obtain in a relatively easy way the rate of decay of the Fourier based distance.

Let us consider a binary interaction between particles governed by the law:

$$
v^{*}=v+n(\lambda v+\mu w), \quad w^{*}=w+n(\lambda w+\mu v) ;
$$

where $(v, w)$ are the pre-collisional velocities which generate the post-collisional ones $\left(v^{*}, w^{*}\right)$. In (3.1) $\lambda$ and $\mu$ are positive constants and $n$ is a parameter which varies in $[-1,1]$.

Let $f(v, t)$ denote the distribution of particles with velocity $v \in \mathbb{R}$ at time $t \geq 0$. A kinetic model governed by binary collisions among particles can be easily derived by standard methods of kinetic theory, considering that the change in time of $f(v, t)$ depends on a balance between the gain and loss of particles with velocity $v$ due to collisions. This leads to the following integro-differential equation of Boltzmann type [25],

$$
\frac{\partial f}{\partial t}=\int_{-1}^{1} \int_{\mathbb{R}} B_{\epsilon}(n)\left(\frac{1}{J} f\left(v_{*}\right) f\left(w_{*}\right)-f(v) f(w)\right) d w d n
$$


In $(3.2)\left(v_{*}, w_{*}\right)$ are the pre-collisional velocities that generate the couple $(v, w)$ after the interaction. $J$ is the Jacobian of the transformation of $(v, w)$ into $\left(v^{*}, w^{*}\right)$, while $B_{\epsilon}(n)$ represents the collision frequency. The collision frequency depends on a small parameter $\epsilon>0$. As $\epsilon \rightarrow 0$, binary collisions concentrate on collisions which are grazing, namely the post-collision velocities are close to the pre-collision ones. This phenomenon is well-known for the Boltzmann equation, where the grazing limit allows to recover in the limit the Landau equation [32], [33]. In what follows, we will assume that $B_{\epsilon}(n)$ satisfies the following properties:

P.1 For all $\epsilon, B_{\epsilon}(n)$ is a symmetric function of $n$, so that $\int_{-1}^{1} B_{\epsilon}(n) n d n=0$

P.2 For all $\epsilon$, the second moment of $B_{\epsilon}(n)$ is bounded, $A_{\epsilon}:=\int_{-1}^{1} B_{\epsilon}(n) n^{2} d n$. Moreover $\lim _{\epsilon \rightarrow 0} A_{\epsilon}=1$

P.3 For any $r>2$, with $r \in \mathbb{N}$, it holds $\lim _{\epsilon \rightarrow 0} \int_{-1}^{1}|n|^{r} B_{\epsilon}(n) d n=0$.

The kinetic equation (3.2) is the analogous of the Boltzmann equation for Maxwell molecules [5]. Also, it presents several similarities with the one-dimensional Kac model without cut-off introduced by Desvillettes [16]. Among the possible choices of the collision frequency, one can consider the following

$$
B_{\epsilon}(n)= \begin{cases}\frac{1}{2 \epsilon\left(n^{2}+\epsilon^{4}\right)} & \text { if } n \in[-\epsilon, \epsilon] \\ 0 & \text { otherwise. }\end{cases}
$$

When necessary, we will denote by $f_{\epsilon}(v, t)$ the solution to the initial value problem for equation (3.2). In this way, we emphasize the dependence of this solution on the $\epsilon$-parameter. Moreover, without loss of generality, we can fix the nonnegative initial density $f_{0}(v)$ to satisfy conditions (1.10).

REMARK 3.1. At a first view, the choice of a nonnegative initial density satisfying conditions (1.10), while quite natural for the study of a kinetic model of Boltzmann type, appears quite restrictive at least for the study of the linearized equation (2.14), where the solution is a perturbation of the Barenblatt profile, and there are no reasons to pretend the perturbation to be positive. To clarify why it is enough to get results on the kinetic equation (3.2) with nonnegative initial data, consider that thanks to mass conservation, this equation can be rewritten as

$$
\frac{\partial f}{\partial t}=Q_{\epsilon}(f, f)-C_{\epsilon} f(v)
$$

where

$$
Q_{\epsilon}(f, f)(v)=\int_{-1}^{1} \int_{\mathbb{R}} B_{\epsilon}(n) \frac{1}{J} f\left(v_{*}\right) f\left(w_{*}\right) d w d n,
$$

and

$$
C_{\epsilon}=\int_{-1}^{1} B_{\epsilon}(n) d n
$$

The solution to equation (3.3) can be expressed as

$$
f(v, t)=f_{0}(v) \exp \left\{-C_{\epsilon} t\right\}+\int_{0}^{t} Q_{\epsilon}(f, f)(v, s) \exp \left\{-C_{\epsilon}(t-s)\right\} d s .
$$


Of course, representation (3.6) holds independently of the sign of the initial datum $f_{0}(v)$. On the other hand, in consequence of the form of the bilinear operator $Q_{\epsilon}$,

$$
|f(v, t)| \leq\left|f_{0}(v)\right| \exp \left\{-C_{\epsilon} t\right\}+\int_{0}^{t} Q_{\epsilon}(|f|,|f|)(v, s) \exp \left\{-C_{\epsilon}(t-s)\right\} d s .
$$

Since the solution to the Boltzmann equation (3.3) is nonnegative in correspondence to a nonnegative initial density, choosing $\left|f_{0}(x)\right|$ as initial density, the solution $f_{+}(v, t)$ to equation (3.3) is nonnegative, and

$$
f_{+}(v, t)=\left|f_{0}(v)\right| \exp \left\{-C_{\epsilon} t\right\}+\int_{0}^{t} Q_{\epsilon}\left(f_{+}, f_{+}\right)(v, s) \exp \left\{-C_{\epsilon}(t-s)\right\} d s .
$$

This implies $|f(v, t)| \leq f_{+}(v, t)$. Thus, any upper bound related to the absolute value of the solution to the Boltzmann equation with a general initial value (with no sign!) can be derived from the same equation taking as initial value a nonnegative density (the absolute value of the initial value). A further comment is in order. Since equation (2.14) is a linear equation on can split the solution in its positive and negative part, and solve the equation for these parts separately. Then the original solution can be recovered by the sum of both.

\subsection{A related conservative kinetic model}

Thanks to the remark of the previous section, without loss of generality, in what follows we will work with nonnegative initial values, satisfying conditions (1.10). To avoid the presence of the Jacobian, and to study approximation to the collision operator it is extremely convenient to write equation (3.2) in weak form. It corresponds to consider, for all smooth functions $\phi(v)$, the equation

$$
\frac{d}{d t} \int_{\mathbb{R}} \phi(v) f_{\epsilon}(v, t) d v=\int_{-1}^{1} \int_{\mathbb{R}^{2}} B_{\epsilon}(n)\left(\phi\left(v^{*}\right)-\phi(v)\right) f_{\epsilon}(v) f_{\epsilon}(w) d w d v d n,
$$

One can alternatively use the symmetric form

$$
\begin{aligned}
\frac{d}{d t} \int_{I R} f_{\epsilon}(v) \phi(v) d v= & \frac{1}{2} \int_{-1}^{1} \int_{\mathbb{R}^{2}} B_{\epsilon}(n) f_{\epsilon}(v) f_{\epsilon}(w) \\
& \left(\phi\left(v^{*}\right)+\phi\left(w^{*}\right)-\phi(v)-\phi(w)\right) d v d w d n .
\end{aligned}
$$

Existence of solutions to (3.9) or, equivalently, to (3.10), can be proven by using arguments like in [16]. Evolution of moments then follows by a suitable choice of the function $\phi$. If $\phi=1$ we obtain from (3.9)

$$
\frac{d}{d t} \int_{\mathbb{R}} f_{\epsilon}(v, t) d v=0
$$

namely mass conservation. Conservation of momentum is obtained by taking $\phi(v)=v$, and making use of assumption P.1. We have

$$
\begin{gathered}
\frac{d}{d t} \int_{\mathbb{R}} v f_{\epsilon}(v, t) d v=\int_{-1}^{1} \int_{\mathbb{R}^{2}} B_{\epsilon}(n)(n(\lambda v+\mu w)) f_{\epsilon}(v) f_{\epsilon}(w) d w d v d n= \\
(\lambda+\mu) \int_{-1}^{1} B_{\epsilon}(n) n d n \int_{\mathbb{R}} v f_{\epsilon}(v) d v=0 .
\end{gathered}
$$


Taking finally $\phi(v)=v^{2}$ we obtain

$$
\begin{gathered}
\frac{d}{d t} \int_{\mathbb{R}} v^{2} f_{\epsilon}(v, t) d v= \\
\int_{-1}^{1} \int_{\mathbb{R}^{2}} B_{\epsilon}(n)\left((v+n(\lambda v+\mu w))^{2}-v^{2}\right) f_{\epsilon}(v) f_{\epsilon}(w) d w d v d n= \\
\int_{-1}^{1} \int_{\mathbb{R}^{2}} B_{\epsilon}(n)\left(n^{2}(\lambda v+\mu w)^{2}+2 n v(\lambda v+\mu w)\right) f_{\epsilon}(v) f_{\epsilon}(w) d w d v d n= \\
\left(\lambda^{2}+\mu^{2}\right) \int_{-1}^{1} B_{\epsilon}(n) n^{2} d n \int_{\mathbb{R}} v^{2} f_{\epsilon}(v) d v=\left(\lambda^{2}+\mu^{2}\right) A_{\epsilon} \int_{\mathbb{R}} v^{2} f_{\epsilon}(v) d v .
\end{gathered}
$$

Since the initial density satisfies (1.10), we obtain that the second moment is exponentially increasing, and

$$
M_{2}(t)=\exp \left\{A_{\epsilon}\left(\lambda^{2}+\mu^{2}\right) t\right\} .
$$

Due to (3.14), it follows clearly that stationary solutions of finite energy do not exist, and the large-time behavior of the kinetic equation can at best be described by selfsimilar solutions. The standard way to look for self-similar profiles is to scale the solution according to the role

$$
g_{\epsilon}(v, t)=\sqrt{M_{2}(t)} f_{\epsilon}\left(v \sqrt{M_{2}(t)}, t\right) .
$$

This scaling implies that $\int v^{2} g_{\epsilon}(v, t)=1$ for all $t \geq 0$. Assuming $\phi$ smooth and of bounded support, by elementary computations one concludes that $g_{\epsilon}=g_{\epsilon}(v, t)$ satisfies the equation

$$
\begin{gathered}
\frac{d}{d t} \int_{\mathbb{R}} \phi(v) g_{\epsilon}(v, t) d v+\frac{\left(\lambda^{2}+\mu^{2}\right) A_{\epsilon}}{2} \int_{\mathbb{R}} v g_{\epsilon} \phi^{\prime}(v) d v= \\
\int_{-1}^{1} \int_{\mathbb{R}^{2}} B_{\epsilon}(n)\left(\phi\left(v^{*}\right)-\phi(v)\right) g_{\epsilon}(v) g_{\epsilon}(w) d w d v d n .
\end{gathered}
$$

Let us consider a third-order Taylor expansion of $\phi\left(v^{*}\right)$ around $v$

$$
\phi\left(v^{*}\right)-\phi(v)=\phi^{\prime}(v)\left(v^{*}-v\right)+\frac{\phi^{\prime \prime}(v)}{2}\left(v^{*}-v\right)^{2}+\frac{\phi^{\prime \prime \prime}(\tilde{v})}{3 !}\left(v^{*}-v\right)^{3}
$$

or equivalently

$$
\phi\left(v^{*}\right)-\phi(v)=\phi^{\prime}(v)(n(\lambda v+\mu w))+\frac{\phi^{\prime \prime}(v)}{2} n^{2}(\lambda v+\mu w)^{2}+\frac{\phi^{\prime \prime \prime}(\tilde{v})}{3 !} n^{3}(\lambda v+\mu w)^{3},
$$

where $\tilde{v}$ is a value between $v$ and $v^{*}$. Substituting the Taylor expansion into the integral on the right-hand side of (3.16), taking the limit $\epsilon \rightarrow 0$ and using property P.3 we obtain that the integral containing the third order term vanishes. Moreover, 
provided $g_{\epsilon}(v, t)$ converges to $g(v, t)$ as $\epsilon \rightarrow 0$, using mass and momentum conservation into the weak form $(3.16)$ we obtain that $g(v, t)$ satisfies

$$
\frac{d}{d t} \int_{\mathbb{R}} \phi(v) g(v, t) d v+\frac{\left(\lambda^{2}+\mu^{2}\right)}{2} \int_{\mathbb{R}} v g \phi^{\prime}(v) d v=\int_{\mathbb{R}} \frac{\left(\lambda^{2} v^{2}+\mu^{2}\right)}{2} \phi^{\prime \prime}(v) g(v) d v,
$$

which is the weak formulation of the following Fokker-Planck equation

$$
\frac{\partial}{\partial t} g(v, t)-\frac{\lambda^{2}+\mu^{2}}{2} \frac{\partial}{\partial v}(v g)=\frac{\partial^{2}}{\partial v^{2}}\left(\frac{\lambda^{2} v^{2}+\mu^{2}}{2} g(v)\right) .
$$

Since in our linearized equations the coefficient in the convection term is one we take $\lambda$ and $\mu$ such that $\lambda^{2}+\mu^{2}=2$. Within this choice,

$$
\frac{\partial}{\partial t} g(v, t)=\frac{\partial}{\partial v}(v g)+\frac{1}{2} \frac{\partial^{2}}{\partial v^{2}}\left(\left(\lambda^{2} v^{2}+\mu^{2}\right) g\right) .
$$

Note that equation (3.19) is nothing but the Fokker-Planck equation (2.1), where

$$
\beta^{2}=\frac{\lambda^{2}}{2} \quad \text { and } \quad \alpha^{2}=\frac{\mu^{2}}{2} .
$$

The passage to the limit outlined above is largely formal. The derivation, however, can be made rigorous by using the same arguments like in [25]. Consider in fact the class of probability densities $\left\{g_{\epsilon}(v, t)\right\}_{\epsilon>0}$. For all $\epsilon$, the solution to (3.16) satisfies conditions (1.10). By virtue of Prokhorov theorem (cfr. [22]) the existence of a uniform bound on the second moment implies that this class is tight, so that any sequence $\left\{g_{\epsilon_{n}}(v, t)\right\}_{n \geq 0}$ contains an infinite subsequence which converges weakly to some probability measure $g(v, t)$. Therefore, the weak solution to the Boltzmann equation (3.16) converges, up to extraction of a subsequence, to a probability density $g(v, t)$. This density is a weak solution of the Fokker-Planck equation (3.19).

\subsection{The evolution of higher moments}

A detailed calculation, along the lines of [25], allows to recognize how many moments of the solution to (3.2) remain uniformly bounded in time with respect to $\epsilon$.

Suppose that the initial density $g_{0}(v)=f_{0}(v)$ is such that

$$
\int_{I R}|v|^{2+\delta} g_{0}(v) d v=M_{2+\delta}<\infty \text {. }
$$

Then, since the contribution due to the term $\frac{\partial}{\partial v}\left(v g_{\epsilon}(v)\right)$ can be evaluated integrating by parts,

$$
\int_{I R}|v|^{2+\delta} \frac{\partial}{\partial v}\left(v g_{\epsilon}(v)\right) d v=-(2+\delta) \int_{I R}|v|^{2+\delta} g_{\epsilon}(v, t) d v
$$

we obtain

$$
\begin{array}{r}
\frac{d}{d t} \int_{I R}|v|^{2+\delta} g_{\epsilon}(v, t) d v+(2+\delta) \frac{\lambda^{2}+\mu^{2}}{2} A_{\epsilon} \int_{I R}|v|^{2+\delta} g_{\epsilon}(v, t) d v= \\
\int_{-1}^{1} B_{\epsilon}(n) d n \int_{I R^{2}} d v d w\left(|(1+\lambda n) v+\mu n w|^{2+\delta}-|v|^{2+\delta}\right) g_{\epsilon}(v) g_{\epsilon}(w) .
\end{array}
$$


Let us recover a suitable upper bound for the last integral in (3.22). Given any two constants $a, b$, and $0<\delta \leq 1$ the following inequality holds

$$
(|a|+|b|)^{\delta} \leq|a|^{\delta}+|b|^{\delta}
$$

Hence, choosing $a=|1+\lambda n||v|$ and $b=|\mu n||w|$,

$$
|(1+\lambda n) v+\mu n w|^{2+\delta} \leq((1+\lambda n) v+\mu n w)^{2}\left(|1+\lambda n|^{\delta}|v|^{\delta}+|\mu n|^{\delta}|w|^{\delta}\right) .
$$

Substituting into the right-hand side of (3.22), recalling that the mean value of $g$ is equal to zero, and the second moment of $g$ equal to one, gives

$$
\begin{gathered}
\int_{-1}^{1} B_{\epsilon}(n) d n \int_{I R^{2}}|(1+\lambda n) v+\mu n w|^{2+\delta} g_{\epsilon}(v) g_{\epsilon}(w) d v d w \leq \\
\int_{I R^{2}}((1+\lambda n) v+\mu n w)^{2}\left(|1+\lambda n|^{\delta}|v|^{\delta}+|\mu n|^{\delta}|w|^{\delta}\right) g_{\epsilon}(v) g_{\epsilon}(w) d v d w= \\
\int_{-1}^{1} B_{\epsilon}(n) d n\left(|1+\lambda n|^{2+\delta}+|\mu n|^{2+\delta}\right) \int_{I R}|v|^{2+\delta} g_{\epsilon}(v) d v+ \\
\int_{-1}^{1} B_{\epsilon}(n) d n\left(|1+\lambda n|^{2}|\mu n|^{\delta}+|\mu n|^{2}|1+\lambda n|^{\delta}\right) \int_{I R}|v|^{\delta} g_{\epsilon}(v) d v
\end{gathered}
$$

Grouping all these inequalities, we obtain

$$
\frac{d}{d t} \int_{I R}|v|^{2+\delta} g_{\epsilon}(v, t) d v \leq \mathcal{S}_{\epsilon}(\delta) \int_{I R}|v|^{2+\delta} g_{\epsilon}(v, t) d v+D_{\delta}
$$

where

$$
\mathcal{S}_{\epsilon}(\delta)=\int_{-1}^{1} B_{\epsilon}(n) d n\left(|1+\lambda n|^{2+\delta}+|\mu n|^{2+\delta}-1\right)-(2+\delta) \frac{\lambda^{2}+\mu^{2}}{2} A_{\epsilon},
$$

and, by Hölder inequality

$$
D_{\delta} \leq \int_{-1}^{1} B_{\epsilon}(n) d n\left(|1+\lambda n|^{2}|\mu n|^{\delta}+|\mu n|^{2}|1+\lambda n|^{\delta}\right) .
$$

By property $\mathbf{P} 3$ it follows that $D_{\delta}$ is uniformly bounded with respect to $\epsilon$. On the other hand, using a Taylor development up to the order three we obtain

$$
|1+\lambda n|^{2+\delta}=1+(2+\delta) \lambda n+\frac{(2+\delta)(1+\delta)}{2} \lambda^{2} n^{2}+O\left(n^{3}\right) .
$$

Substituting into (3.25) we obtain

$$
\mathcal{S}_{\epsilon}=\frac{2+\delta}{2}\left(\lambda^{2} \delta-\mu^{2}\right) A_{\epsilon}+0(\epsilon) .
$$

Hence, $\mathcal{S}_{\epsilon} \leq 0$ as soon as $\lambda^{2} \delta-\mu^{2}<0$ and $\epsilon$ is suitably small. In this case, inequality (3.24) gives an upper bound for the moment, that reads

$$
\int_{I R}|v|^{2+\delta} g_{\epsilon}(v, t) d v \leq M_{2+\delta}+\frac{D_{\delta}}{\left|\mathcal{S}_{\epsilon}(\delta)\right|}<\infty
$$


Let us set $\bar{\delta}=\mu^{2} / \lambda^{2}$. In the case $\bar{\delta}>3$ we can easily iterate our procedure to obtain that any moment of order $2+\delta$, with $\delta<\bar{\delta}$ which is bounded initially, remains uniformly bounded with respect to $\epsilon$ at any subsequent time. The only difference now is that the explicit expression of the bound is more and more involved. We proved LEMMA 3.1. Let $g_{\epsilon}(v, t)$ be the solution to the initial value problem for the kinetic equation (3.16), where the initial datum $g_{0}(v)$ satisfies

$$
\int_{I R}|v|^{2+\delta} g_{0}(v) d v=M_{2+\delta}<\infty .
$$

Then, if $\delta<\bar{\delta}=\mu^{2} / \lambda^{2}$,

$$
\int_{I R}|v|^{2+\delta} g_{\epsilon}(v, t) d v
$$

is uniformly bounded in time for all $\epsilon$. Moreover, if $\delta<\bar{\delta}$ the function $\mathcal{S}_{\epsilon}$ defined by (3.25) satisfies

$$
\lim _{\epsilon \rightarrow 0} \mathcal{S}_{\epsilon}(\delta)<0
$$

\subsection{Rate of convergence in Fourier based metrics}

Let $\mathcal{M}_{0}$ denote the space of all probability measures in $\mathbb{R}$ and

$$
\mathcal{M}_{r}=\left\{\mu \in \mathcal{M}_{0}: \int_{\mathbb{R}}|v|^{r} \mu(d v)<\infty, r \geq 0\right\}
$$

the space of all Borel probability measures with finite momentum of order $r$ equipped with the topology of the weak convergence of the measures. By a weak solution of the initial value problem for equation (3.2), corresponding to the initial probability density $f_{0}(w) \in \mathcal{M}_{r} r>2$ we shall mean any probability density $f \in C^{1}\left(\mathbb{R}, \mathcal{M}_{r}\right)$ satisfying the weak form of the equation (3.9) for $t>0$ and all smooth functions $\phi$, and such that for all $\phi$

$$
\lim _{t \rightarrow 0} \int_{I R} \phi(v) f(v, t) d v=\int_{I R} \phi(v) f_{0}(v) d v .
$$

In the rest of this section, we shall study the weak form of equation (3.2), with the normalization conditions (1.10). It is equivalent to use the Fourier transform of the equation [5]:

$$
\frac{\partial \widehat{f}(\xi, t)}{\partial t}=\widehat{Q}(\widehat{f}, \widehat{f})(\xi, t)
$$

where $\widehat{f}(\xi, t)$ is the Fourier transform of $f(v, t)$ and

$$
\widehat{Q}(\widehat{f}, \widehat{f})(\xi)=\int_{-1}^{1} B_{\epsilon}(n)[\hat{f}(\xi(1+\lambda n), t) \hat{f}(n \mu \xi, t)-\hat{f}(\xi, t)] d n .
$$

The initial conditions (1.10) turn into

$$
\widehat{f}(0)=1, \widehat{f}^{\prime}(0)=0, \widehat{f}^{\prime \prime}(0)=-1,
$$


where $\widehat{f}_{0} \in C^{2}(\mathbb{R})$. Hence equation (3.34) can be rewritten (recall (3.5)) as

$$
\frac{\partial \widehat{f}(\xi, t)}{\partial t}+C_{\epsilon} \widehat{f}(\xi, t)=\int_{-1}^{1} B_{\epsilon}(n) \hat{f}(\xi(1+\lambda n), t) \hat{f}(n \mu \xi, t) d n .
$$

We work with the metric introduced in [19]

$$
d_{s}(f, g)=\sup _{\xi \in \mathbb{R}} \frac{|\hat{f}(\xi)-\hat{g}(\xi)|}{|\xi|^{s}} .
$$

It is easy to show that (see [26], [25]), for any given collision integral of form (3.35), given two initial densities $f_{0,1}$ and $f_{0,2}$ belonging to $\mathcal{M}_{r}, r>2$, and such that $d_{r}\left(f_{0,1}, f_{0,2}\right)$ is bounded, $d_{r}\left(f_{1}(t), f_{2}(t)\right)$ is bounded at any subsequent time $t>0$, and the following inequality holds

$$
\begin{gathered}
d_{r}\left(f_{1}(t), f_{2}(t)\right) \leq d_{r}\left(f_{0,1}, f_{0,2}\right) . \\
\cdot \exp \left\{\left(\int_{-1}^{1} B_{\epsilon}(n)\left[|1+\lambda n|^{r}+(\mu n)^{r}-1\right] d n\right) t\right\} .
\end{gathered}
$$

Inequality (3.38) implies a similar inequality for the scaled function $g$, which we recall is defined through the scaling $g(v, t)=\sqrt{M_{2}(t)} f\left(v \sqrt{M_{2}(t)}, t\right)$. The Fourier transform of $g$ is $\hat{g}(\xi)=\hat{f}\left(\xi / \sqrt{M_{2}(t)}\right)$. From the definition of $d_{r}$ it follows

$$
d_{r}\left(g_{1}(t), g_{2}(t)\right)=\left(\frac{1}{\sqrt{M_{2}(t)}}\right)^{r} d_{r}\left(f_{1}(t), f_{2}(t)\right) .
$$

Therefore

$$
\begin{gathered}
d_{r}\left(g_{1}(t), g_{2}(t)\right) \leq d_{r}\left(g_{0,1}, g_{0,2}\right) . \\
\cdot \exp \left\{\left(\int_{-1}^{1} B_{\epsilon}(n)\left[|1+\lambda n|^{r}+|\mu n|^{r}-1-\frac{r}{2} n^{2}\left(\lambda^{2}+\mu^{2}\right)\right] d n\right) t\right\} .
\end{gathered}
$$

The exponent in (3.39) coincides with the function $\mathcal{S}_{\epsilon}(\cdot)$ defined in $(3.25)$, and evaluated at $\delta=r-2$.

Applying Lemma 2.2, and taking the limit $\epsilon \rightarrow 0$ on (3.39) and calling again $g$ the solution, for all $\delta<r-2$ we find that the solution to the Fokker-Planck equation (3.18) satisfies

$$
\left.d_{2+\delta}\left(g_{1}(t), g_{2}(t)\right) \leq d_{2+\delta}\left(g_{0,1}, g_{0,2}\right) \exp \left\{\frac{2+\delta}{2}\left(\lambda^{2} \delta-\mu^{2}\right)\right) t\right\}
$$

The exact rate of convergence in $\delta$-metric follows by analyzing the parabola $h(\delta)=$ $\frac{2+\delta}{2}\left(\lambda^{2} \delta-\mu^{2}\right)$, that is nonpositive in the interval $\delta \in\left[0, \bar{\delta}=\mu^{2} / \lambda^{2}\right]$ (see Figure ??). The minimum value in this interval is achieved at $\delta_{0}=\bar{\delta} / 2-1$ where $h\left(\delta_{0}\right)=$ $-\frac{1}{8}\left(\mu^{2}+2 \lambda^{2}\right)$. Thus, if $\delta_{0}>0$, in the interval $\delta \in\left[0, \delta_{0}\right]$ the rate of convergence in 
$d_{2+\delta}$-metric is increasing, and we have a gain in the rate of convergence if the initial data have a big number of equal moments. Now, $\delta_{0}>0$ if $\mu^{2}>2 \lambda^{2}$, or, what is the same (see $(3.20))$,

$$
\alpha^{2}=\frac{\mu^{2}}{2}>2 \beta^{2}=\frac{2 \lambda^{2}}{2} .
$$

This implies that we have an improved rate of convergence in $d_{2+\delta}$-metric for equation (2.18) in the range $1 / 3<m<1$, while the same holds for equation (2.21) in the range $3 / 5<m<1$. We include these results into the following.

THEOREM 3.2. Let $f(v, t)$ be the solution to equation (2.14), corresponding to the initial datum $f_{0}(v)$ satisfying (1.10). Suppose in addition that

$$
d_{2+\delta}\left(f_{0}, D B_{C}^{2-m}\right)<+\infty, \quad \delta<(1+m) /(1-m) .
$$

Then, the solution $f(v, t)$ converges exponentially to $B_{C}(v)^{2-m}$ in $d_{2+\delta}-m e t r i c$, and

$$
d_{2+\delta}\left(f(t), D B_{C}^{2-m}\right) \leq d_{2+\delta}\left(f_{0}, D B_{C}^{2-m}\right) \exp \left\{\frac{2+\delta}{2}((1-m) \delta-(1+m)) t\right\} .
$$

Moreover, provided $1 / 3<m<1$ the rate of convergence in (3.43) increases in the interval $0<\delta \leq(3 m-1) /(2-2 m)$. Likewise, let $f(v, t)$ be the solution to equation (1.6), with initial datum $f_{0}(v)$. Suppose in addition that

$$
d_{2+\delta}\left(f_{0}, B_{C}\right)<+\infty, \quad \delta<(3 m-1) /(1-m) .
$$

Then, the solution $f(v, t)$ converges exponentially to $B_{C}(x)$ in $d_{2+\delta}-m e t r i c$, and

$$
d_{2+\delta}\left(f(t), B_{C}\right) \leq d_{2+\delta}\left(f_{0}, B_{C}\right) \exp \left\{\frac{2+\delta}{2}\left(\frac{1-m}{m} \delta-\frac{3 m-1}{m}\right) t\right\} .
$$

Moreover, provided $3 / 5<m<1$ the rate of convergence in (3.45) increases in the interval $0<\delta \leq(5 m-3) /(2-2 m)$.

Proof. The proof of this theorem is a direct consequence of the previous computations and we can obtain it by identification of coefficients, as follows. First of all, we recall that both linear equation $((2.14)$ and (1.6)) can be written using different scaling as equation (2.1) (see section 2.1). For equation (2.14) we have

$$
\alpha^{2}=\frac{\mu^{2}}{2}=\frac{1+m}{2} \text { and } \quad \beta^{2}=\frac{\lambda^{2}}{2}=\frac{1-m}{2}
$$

thus (3.41) holds if and only if $m>1 / 3$. On the other hand, since

$$
\mu^{2}=1+m \quad \text { and } \quad \lambda^{2}=1-m
$$

we obtain that the rate of convergence $\frac{2+\delta}{2}\left(\lambda^{2} \delta-\mu^{2}\right)$ is written as

$$
\frac{2+\delta}{2}((1-m) \delta-(1+m)) \text {. }
$$

And the minimum value is achieved in $\delta_{0}=\frac{3 m-1}{2-2 m}$ (see previous computations) and this value is

$$
-\frac{1}{2^{3}} \frac{(3-m)^{2}}{1-m}
$$


For equation (1.6) we have

$$
\alpha^{2}=\frac{\mu^{2}}{2}=\frac{3 m-1}{2 m} \quad \text { and } \quad \beta^{2}=\frac{\lambda^{2}}{2}=\frac{1-m}{2 m}
$$

and in this case (3.41) holds if and only if $m>3 / 5$ and the rate of convergence is written as

$$
\frac{2+\delta}{2}\left(\frac{1-m}{2 m} \delta-\frac{3 m-1}{2 m}\right) .
$$

And the minimum value is achieved in $\delta_{0}=\frac{5 m-3}{2-2 m}$ (see previous computations) and this value is

$$
-\frac{1}{2^{3}} \frac{(m+1)^{2}}{m}
$$

$\square$

The result of Theorem 3.2 introduces new bounds into the game. As a matter of fact, while our method of proof improves the rate of convergence in both cases as soon as we are sufficiently close to the linear heat equation, while we are not able to show improvements in the rate of convergence to equilibrium as soon as we are too far from the linear one.

\section{The $N$-dimensional Fokker-Planck equation}

The results of Section 3 clarify the strategy we used to obtain precise rates of convergence towards the steady state for the one-dimensional Fokker-Planck equation (2.4). In one dimension of the velocity variable the connection between nonconservative models of the Boltzmann equation and the Fokker-Planck equation with non constant diffusion coefficients was known for binary collisions of different type [25]. To our knowledge, however, in higher dimensions, at present no studies on this connection have been considered so far. Clearly, higher dimensional models have been studied in the grazing limit in the conservative case by Villani [32] [33], but there, starting from the nonlinear Boltzmann equation with elastic collisions, the limit equation results in the nonlinear Landau equation. To obtain in the limit a linear Fokker-Planck type equation requires the introduction of a particular collision dynamics. We deal with this extension in the forthcoming Section. Since many arguments will be taken from the one-dimensional analysis of Section 3, when not necessary to the understanding, we will only briefly outline them.

\section{1. $N$-dimensional binary collisions and nonconservative kinetic mod-} els

The starting point of our analysis will be the introduction of a particular collision dynamics, that in the grazing limit is able to produce the target equation (2.4). Clearly, as the one-dimensional analysis suggests, this is not the only possible model of binary collision that produces the correct result. Nevertheless, this law is in our opinion the simplest possible generalization of (3.1) to higher dimensions. The binary interaction between particles will be governed by the law:

$$
v^{*}=v+n(\lambda e \cdot v+\mu e \cdot w), \quad w^{*}=w+n(\lambda e \cdot w+\mu e \cdot v) .
$$

In (4.1) $(v, w) \in \mathbb{R}^{2 N}$ are the pre-collisional velocities which generate the postcollisional ones $\left(v^{*}, w^{*}\right) \in \mathbb{R}^{2 N}, \lambda$ and $\mu$ are positive constants, $n \in[-1,1]^{N}$ and $e \in \mathbb{R}^{N}$ 
is a unit vector. Consequently, the integro-differential equation of Boltzmann type (3.2) is written as

$$
\frac{\partial f}{\partial t}=\int_{C_{N}} \int_{S^{N-1}} \int_{\mathbb{R}^{N}} \frac{B_{\epsilon}(n)}{B_{N}}\left(\frac{1}{J} f\left(v_{*}\right) f\left(w_{*}\right)-f(v) f(w)\right) d w d e d n
$$

where $\left(v_{*}, w_{*}\right)$ are the pre-collisional velocities that generate the couple $(v, w)$ after the interaction, $J$ is the Jacobian of the transformation of $(v, w)$ into $\left(v^{*}, w^{*}\right), C_{N}$ denotes the cube in $\mathbb{R}^{N}$ centered on the origin with dimensions $[-1,1]^{N}, S^{N-1}$ represents the unit sphere in $\mathbb{R}^{N}, B_{N}$ is the measure of the surface of $S^{N-1}$ and $B_{\epsilon}(n)$ represents the collision frequency. Proceedings as in dimension one, we will assume that $B_{\epsilon}(n)$ satisfies the following properties:

$\mathbf{P}_{\mathrm{N}}$.1 For all $\epsilon, B_{\epsilon}(n)$ is a symmetric function of all the components of $n$, so that

$$
\text { for all } i \int_{C_{N}} B_{\epsilon}(n) n_{i} d n=0, \quad \text { for all } i \neq j, \int_{C_{N}} B_{\epsilon}(n) n_{i} n_{j} d n=0
$$

$\mathbf{P}_{\mathbf{N}} \cdot \mathbf{2}$ For all $\epsilon$, the second moment of $B_{\epsilon}(n)$ is bounded,

$$
A_{\epsilon}:=\int_{C_{N}} B_{\epsilon}(n)|n|^{2} d n<\infty \text {. Moreover } \lim _{\epsilon \rightarrow 0} A_{\epsilon}=1 \text {. }
$$

$\mathbf{P}_{\mathbf{N}} .3$ For any $r>2$, with $r \in \mathbb{N}$, it holds $\lim _{\epsilon \rightarrow 0} \int_{C_{N}}|n|^{r} B_{\epsilon}(n) d n=0$.

The weak formulation of (4.2) now reads

$$
\begin{aligned}
\frac{d}{d t} \int_{\mathbb{R}^{N}} f_{\epsilon}(v) \phi(v) d v= & \frac{1}{2} \int_{C_{N}} \int_{S^{N-1}} \int_{\mathbb{R}^{2 N}} \frac{B_{\epsilon}(n)}{B_{N}} f_{\epsilon}(v) f_{\epsilon}(w) \\
& \left(\phi\left(v^{*}\right)+\phi\left(w^{*}\right)-\phi(v)-\phi(w)\right) d v d w d e d n
\end{aligned}
$$

Conservation of mass follows directly considering $\phi(v)=1$ and using $\mathbf{P}_{\mathbf{N}}$.1. Likewise, conservation of momentum follows by taking $\phi(v)=v$ and using $\mathbf{P}_{\mathbf{N}} \cdot \mathbf{1}$ again. The evaluation of the law of variation of the second momentum requires computations we present below. Precisely we obtain

LEMMA 4.1. Let $f_{\epsilon}$ be a solution of (4.2) corresponding to an initial value satisfying conditions (1.10). Then the moment of order two is exponentially increasing and it holds

$$
M_{2}(t)=\exp \left\{\frac{A_{\epsilon}\left(\lambda^{2}+\mu^{2}\right)}{N} t\right\}
$$

Proof. Choosing $\phi(v)=|v|^{2}$ into the weak form (4.3) we find

$$
\begin{aligned}
\frac{d}{d t} \int_{\mathbb{R}^{N}} f_{\epsilon}(v)|v|^{2} d v= & \frac{1}{2} \int_{C_{N}} \int_{S^{N-1}} \int_{\mathbb{R}^{2 N}} \frac{B_{\epsilon}(n)}{B_{N}} f_{\epsilon}(v) f_{\epsilon}(w) . \\
& \cdot\left(\left|v^{*}\right|^{2}+\left|w^{*}\right|^{2}-|v|^{2}-|w|^{2}\right) d v d w d e d n .
\end{aligned}
$$

By the collision rule (4.1) we obtain

$$
\begin{aligned}
& \frac{d}{d t} \int_{\mathbb{R}^{N}} f_{\epsilon}(v)|v|^{2} d v=\int_{C_{N}} \int_{S^{N-1}} \int_{\mathbb{R}^{2 N}} \frac{B_{\epsilon}(n)}{B_{N}} f_{\epsilon}(v) f_{\epsilon}(w) \cdot \\
& \cdot\left(|n|^{2}|\lambda e \cdot v+\mu e \cdot w|^{2}+2(\lambda e \cdot v+\mu e \cdot w) v \cdot n\right) d v d w d e d n .
\end{aligned}
$$


By property $\mathbf{P}_{\mathbf{N}} \cdot \mathbf{1}$ follows

$$
\int_{C_{N}} \int_{S^{N-1}} \int_{\mathbb{R}^{2 N}} \frac{B_{\epsilon}(n)}{B_{N}} f_{\epsilon}(v) f_{\epsilon}(w)(\lambda e \cdot v+\mu e \cdot w) v \cdot n d v d w d e d n=0 .
$$

Therefore,

$$
\begin{aligned}
& \frac{d}{d t} \int_{\mathbb{R}^{N}} f_{\epsilon}(v)|v|^{2} d v=\int_{C_{N}} \int_{S^{N-1}} \int_{\mathbb{R}^{2 N}} \frac{B_{\epsilon}(n)}{B_{N}} f_{\epsilon}(v) f_{\epsilon}(w) . \\
& \cdot|n|^{2}|\lambda e \cdot v+\mu e \cdot w|^{2} d v d w d e d n
\end{aligned}
$$

which can be written as

$$
\begin{aligned}
\frac{d}{d t} \int_{\mathbb{R}^{N}} f_{\epsilon}(v)|v|^{2} d v= & \int_{C_{N}} \int_{S^{N-1}} \int_{\mathbb{R}^{2 N}} \frac{B_{\epsilon}(n)}{B_{N}} f_{\epsilon}(v) f_{\epsilon}(w) \\
& |n|^{2}\left(\lambda^{2}(e \cdot v)^{2}+\mu^{2}(e \cdot w)^{2}+2 \lambda \mu(e \cdot v)(e \cdot w)\right) d v d w d e d n .
\end{aligned}
$$

Finally, since mass and momentum are conserved, we obtain

$$
\begin{aligned}
\frac{d}{d t} \int_{\mathbb{R}^{N}} f_{\epsilon}(v)|v|^{2} d v & =\int_{C_{N}} \int_{S^{N-1}} \int_{\mathbb{R}^{2 N}} \frac{B_{\epsilon}(n)}{B_{N}} f_{\epsilon}(v) f_{\epsilon}(w) \\
& |n|^{2}\left(\lambda^{2}(e \cdot v)^{2}+\mu^{2}(e \cdot w)^{2}\right) d v d w d e d n \\
& =\left(\lambda^{2}+\mu^{2}\right) \int_{C_{N}} \int_{S^{N-1}} \int_{\mathbb{R}^{N}} \frac{B_{\epsilon}(n)}{B_{N}} f_{\epsilon}(v)|n|^{2}(e \cdot v)^{2} d v d e d n \\
& =\frac{A_{\epsilon}\left(\lambda^{2}+\mu^{2}\right)}{N} \int_{\mathbb{R}^{N}} f_{\epsilon}(v)|v|^{2} d v
\end{aligned}
$$

The last equality follows by symmetry, since from the equality

$$
\frac{1}{B_{N}} \int_{S^{N-1}} e_{i}^{2} d e=\frac{1}{B_{N}} \int_{S^{N-1}} e_{j}^{2} d e, \quad i \neq j
$$

it follows

$$
\frac{1}{B_{N}} \int_{S^{N-1}} e_{i}^{2} d e=\frac{1}{N} \quad i=1, \ldots n
$$

and

$$
\frac{1}{B_{N}} \int_{S^{N-1}}(e \cdot v)^{2} d e=\frac{1}{N}|v|^{2}
$$

As discussed in the one-dimensional case, the exponential growth of the second moment implies that do not exist stationary solutions with finite energy. To look for self-similar profiles, we scale the solution according to

$$
g_{\epsilon}(v, t)=\left(M_{2}(t)\right)^{N / 2} f_{\epsilon}\left(v \sqrt{M_{2}(t)}, t\right) .
$$

By this scaling, we obtain that the second moment of $g_{\epsilon}$ remains equal to one for all $t \geq 0$. Thus, if the initial value to the kinetic equation (4.2) satisfies condition (1.10), 
so does $g_{\epsilon}(v, t)$ at any subsequent time. The weak formulation of equation (4.2) for the scaled function $g_{\epsilon}$ is written as

$$
\begin{gathered}
\frac{d}{d t} \int_{\mathbb{R}^{N}} \phi(v) g_{\epsilon}(v, t) d v+\frac{\left(\lambda^{2}+\mu^{2}\right) A_{\epsilon}}{2 N} \int_{\mathbb{R}^{N}} \nabla \phi \cdot v g_{\epsilon} d v= \\
\int_{C_{N}} \int_{S^{N-1}} \int_{\mathbb{R}^{2 N}} \frac{B_{\epsilon}(n)}{B_{N}}\left(\phi\left(v^{*}\right)-\phi(v)\right) g_{\epsilon}(v) g_{\epsilon}(w) d w d v d e d n
\end{gathered}
$$

If we consider a third-order Taylor development of $\phi\left(v^{*}\right)$ around $v$ we obtain

$$
\phi\left(v^{*}\right)-\phi(v)=\nabla_{v} \phi(v) \cdot\left(v^{*}-v\right)+\frac{1}{2}\left(v^{*}-v\right) \operatorname{Hess}(\phi)(v)\left(v^{*}-v\right)^{t}+R\left(\phi, \tilde{v}, v^{*}, v\right)
$$

where we denoted by Hess the Hessian matrix of a function, and by $w^{t}$ the transpose of a vector $w$. Moreover $R$ represents the remainder evaluated in correspondence to a vector $\tilde{v}=\lambda v+(1-\lambda) v^{*}$, where $\lambda$ is a suitable constant, $0 \leq \lambda \leq 1$. Resorting to the expression of $v^{*}$ in terms of $v$ we find

$$
\begin{gathered}
\phi\left(v^{*}\right)-\phi(v)=\nabla_{v} \phi(v) \cdot n(\lambda e \cdot v+\mu e \cdot w)+ \\
\frac{1}{2} n(\lambda e \cdot v+\mu e \cdot w) \operatorname{Hess}(\phi)(v)(n(\lambda e \cdot v+\mu e \cdot w))^{t}+R\left(\phi, \tilde{v}, v^{*}, v\right) .
\end{gathered}
$$

Substituting the above expression into (4.7), and owing to property $\mathbf{P}_{\mathbf{N}} \cdot \mathbf{3}$ shows that the term corresponding to the remainder vanishes when $\epsilon$ goes to zero. Therefore, we will focus on the remaining two terms. The integral corresponding to the first term in the Taylor expansion vanishes thanks to property $\mathbf{P}_{\mathbf{N}}$.1. In fact

$$
\begin{gathered}
\int_{C_{N}} \int_{S^{N-1}} \int_{\mathbb{R}^{2 N}} \frac{B_{\epsilon}(n)}{B_{N}} \nabla_{v} \phi(v) \cdot n(\lambda e \cdot v+\mu e \cdot w) g_{\epsilon}(v) g_{\epsilon}(w) d w d v d e d n= \\
\int_{S^{N-1}} \int_{\mathbb{R}^{2 N}} \frac{1}{B_{N}} \nabla_{v} \phi(v) \cdot\left(\int_{C_{N}} B_{\epsilon}(n) n d n\right)(\lambda e \cdot v+\mu e \cdot w) g_{\epsilon}(v) g_{\epsilon}(w) d w d v d e=0 .
\end{gathered}
$$

On the other hand, by property $\mathbf{P}_{\mathbf{N}} \mathbf{1}$ the second term can we written as follow

$$
\begin{gathered}
\frac{1}{2} \int_{C_{N}} \int_{S^{N-1}} \int_{\mathbb{R}^{2 N}} \frac{B_{\epsilon}(n)}{B_{N}} g_{\epsilon}(v) g_{\epsilon}(w) n(\lambda e \cdot v+\mu e \cdot w) \\
\operatorname{Hess}\left(g_{\epsilon}\right)(v)(n(\lambda e \cdot v+\mu e \cdot w))^{t} d w d v d e d n= \\
\frac{1}{2} \int_{S^{N-1}} \int_{\mathbb{R}^{2 N}} \frac{1}{B_{N}} g_{\epsilon}(v) g_{\epsilon}(w)\left(\sum_{i} \frac{\partial^{2} \phi(v)}{\partial v_{i}^{2}} \int_{C_{N}} B_{\epsilon}(n) n_{i}^{2} d n\right) \\
(\lambda e \cdot v+\mu e \cdot w)^{2} d w d v d e=
\end{gathered}
$$




$$
\begin{aligned}
& \frac{1}{2} \int_{S^{N-1}} \int_{\mathbb{R}^{2 N}} \frac{1}{B_{N}} g_{\epsilon}(v) g_{\epsilon}(w)\left(\sum_{i} \frac{\partial^{2} \phi(v)}{\partial v_{i}^{2}} \frac{A_{\epsilon}}{N}\right) \\
& \left(\lambda^{2}(e \cdot v)^{2}+\mu^{2}(e \cdot w)^{2}+2 \lambda \mu(e \cdot v)(e \cdot w)\right) d w d v d e= \\
& \frac{A_{\epsilon}}{2 N B_{N}} \int_{S^{N-1}} \int_{\mathbb{R}^{2 N}} g_{\epsilon}(v) g_{\epsilon}(w) \Delta \phi(v) \\
& \left(\lambda^{2}(e \cdot v)^{2}+\mu^{2}(e \cdot w)^{2}+2 \lambda \mu(e \cdot v)(e \cdot w)\right) d w d v d e= \\
& \frac{A_{\epsilon}}{2 N B_{N}} \int_{S^{N-1}} \int_{\mathbb{R}^{2 N}} g_{\epsilon}(v) g_{\epsilon}(w) \Delta \phi(v)\left(\lambda^{2}(e \cdot v)^{2}+\mu^{2}(e \cdot w)^{2}\right) d w d v d e= \\
& \frac{A_{\epsilon}}{2 N^{2}} \int_{\mathbb{R}^{2 N}} g_{\epsilon}(v) g_{\epsilon}(w) \Delta \phi(v)\left(\lambda^{2}|v|^{2}+\mu^{2}|w|^{2}\right) d w d v= \\
& \frac{A_{\epsilon}}{2 N^{2}} \int_{\mathbb{R}^{N}} g_{\epsilon}(v) \Delta \phi(v)\left(\lambda^{2}|v|^{2}+\mu^{2}\right) d v .
\end{aligned}
$$

Therefore, collecting terms, and taking the limit $\epsilon \rightarrow 0$ we find that the limit function $g(v, t)$ satisfies the equation (in weak form)

$$
\begin{gathered}
\frac{d}{d t} \int_{\mathbb{R}^{N}} \phi(v) g(v, t) d v+\frac{\left(\lambda^{2}+\mu^{2}\right) A_{\epsilon}}{2 N} \int_{\mathbb{R}^{N}} \nabla \phi(v) \cdot v g d v= \\
\frac{A_{\epsilon}}{2 N^{2}} \int_{\mathbb{R}^{N}} \Delta \phi g(v)\left(\lambda^{2}|v|^{2}+\mu^{2}\right) d v
\end{gathered}
$$

which is the weak formulation of the following Fokker-Planck equation

$$
\frac{\partial}{\partial t} g(v, t)-\frac{\lambda^{2}+\mu^{2}}{2 N} \operatorname{div}(v g)=\Delta\left(\frac{\lambda^{2} v^{2}+\mu^{2}}{2 N^{2}} g(v)\right) .
$$

As in the one-dimensional case the coefficient in front of the convection term can be made equal to one by setting $\lambda^{2}+\mu^{2}=2 N$. Within this choice,

$$
\frac{\partial}{\partial t} g(v, t)=\operatorname{div}(v g)+\Delta\left[\left(\frac{\lambda^{2}}{2 N^{2}} v^{2}+\frac{\mu^{2}}{2 N^{2}}\right) g(v)\right] .
$$

Again, as in the one dimensional case, it follows that the limit function $g(v, t)$ satisfies the Fokker-Planck equation (2.1), by taking

$$
\alpha^{2}=\frac{\mu^{2}}{2 N^{2}} \quad \text { and } \quad \beta^{2}=\frac{\lambda^{2}}{2 N^{2}}
$$

which satisfy $\alpha^{2}+\beta^{2}=1 / N$. We can now repeat the reasoning we did in one-dimension of space, to conclude that the sequence $\left\{g_{\epsilon}(v, t)\right\}_{\epsilon \geq 0}$ contains a subsequence that converges weakly to a function $g$ satisfying (4.8).

4.2. Convergence in Fourier based metrics We use the same notation as in one dimensional case and we denote by $\mathcal{M}_{0}$ the space of all probability measures in $\mathbb{R}^{N}$ and

$$
\mathcal{M}_{r}=\left\{\mu \in \mathcal{M}_{0}: \int_{\mathbb{R}^{N}}|v|^{r} \mu(d v)<\infty, r \geq 0\right\}
$$


the space of all Borel probability measures with finite momentum of order $r$ equipped with the topology of the weak convergence of the measures. A weak solution of the initial value problem for equation (4.2), corresponding to the initial probability density $f_{0}(w) \in \mathcal{M}_{r}, r>2$ we shall mean any probability density $f \in C^{1}\left(\mathbb{R}^{N}, \mathcal{M}_{r}\right)$ satisfying the weak form of the equation (4.3) for $t>0$ and all smooth functions $\phi$, and such that for all $\phi$

$$
\lim _{t \rightarrow 0} \int_{I R} \phi(v) f(v, t) d v=\int_{I R} \phi(v) f_{0}(v) d v .
$$

In the rest of this section, we shall study the weak form of equation (4.2), with the normalization conditions (1.10). It is equivalent to use the Fourier transform of the equation $[5]$ :

$$
\frac{\partial \widehat{f}(\xi, t)}{\partial t}=\widehat{Q}(\widehat{f}, \widehat{f})(\xi, t)
$$

where

$$
\begin{aligned}
\widehat{Q}(\widehat{f}, \hat{f})(\xi)= & \int_{C_{N}} \int_{S^{N-1}} \frac{B_{\epsilon}(n)}{B_{N}}[\hat{f}(\xi+(n \cdot \xi) \lambda e, t) \hat{f}((n \cdot \xi) \mu e, t)-\hat{f}(\xi, t)] d e d n \\
& \int_{C_{N}} \int_{S^{N-1}} \frac{B_{\epsilon}(n)}{B_{N}} \hat{f}(\xi+(n \cdot \xi) \lambda e, t) \hat{f}((n \cdot \xi) \mu e, t) d e d n-C_{\epsilon}^{N} \hat{f}(\xi, t)
\end{aligned}
$$

where

$$
C_{\epsilon}^{N}=\int_{C_{N}} B_{\epsilon}(n) d n
$$

In the $N$-dimensional case, the initial conditions (1.10) take the form

$$
\widehat{f_{0}}(\xi=0)=1, \quad \nabla \widehat{f}_{0}(\xi=0)=0, \quad \Delta \widehat{f}_{0}(\xi=0)=-1,
$$

where $\widehat{f}_{0} \in C^{2}\left(\mathbb{R}^{N}\right)$.

Then, proceeding as in the one-dimensional case [19], given two initial data $f_{0,1}$ and $f_{0,2}$ such that $d_{r}\left(f_{0,1}, f_{0,2}\right)$ is bounded for some $r \geq 2$, one obtains for the corresponding solutions to $((4.3))$ the estimate

$$
d_{r}\left(f_{1}(t), f_{2}(t)\right) \leq d_{r}\left(f_{0,1}, f_{0,2}\right) \cdot \exp \left\{R_{N}^{\epsilon}(r) t\right\}
$$

where

$$
R_{N}^{\epsilon}(r)=\int_{C_{N}} \int_{S^{N-1}} \frac{B_{\epsilon}(n)}{B_{N}}\left[|\gamma+(n \cdot \gamma) \lambda e|^{r}+|(n \cdot \gamma) \mu e|^{r}-1\right] d e d n,
$$

and $\gamma=\xi /|\xi|$ denotes a unit vector. The estimation (4.14) is translated to the analogous estimation for the scaled function $g_{\epsilon}$. As in the one-dimensional case

$$
d_{r}\left(g_{\epsilon_{1}}(t), g_{\epsilon_{2}}(t)\right)=M_{2}(t)^{-r / 2} d_{r}\left(f_{1}(t), f_{2}(t)\right) \leq d_{r}\left(f_{0,1}, f_{0,2}\right) \cdot \exp \left\{R_{N}^{\epsilon}(r) t\right\}
$$

or equivalently, since $d_{r}\left(f_{0,1}, f_{0,2}\right)=d_{r}\left(g_{0,1}, g_{0,2}\right)$

$$
d_{r}\left(g_{\epsilon_{1}}(t), g_{\epsilon_{2}}(t)\right)=M_{2}(t)^{-r / 2} d_{r}\left(f_{1}(t), f_{2}(t)\right) \leq d_{r}\left(g_{\epsilon_{01}}, g_{\epsilon_{02}}\right) \cdot \exp \left\{S_{N}^{\epsilon}(r) t\right\}
$$


where now

$$
\begin{gathered}
S_{N}^{\epsilon}(r)=\int_{C_{N}} \int_{S^{N-1}} \frac{B_{\epsilon}(n)}{B_{N}} . \\
\cdot\left[|\gamma+(n \cdot \gamma) \lambda e|^{r}+|(n \cdot \gamma) \mu e|^{r}-1-\frac{r}{2 N} n^{2}\left(\lambda^{2}+\mu^{2}\right)\right] d e d n .
\end{gathered}
$$

In order to pass to the limit $\epsilon \rightarrow 0$ we use a Taylor development for the function $h(v)=|v|^{2+\delta}$ with $v \in \mathbb{R}^{N}$,

$$
h(v)=h\left(v_{0}\right)+\nabla_{v} h\left(v_{0}\right) \cdot\left(v-v_{0}\right)+\frac{1}{2}\left(v-v_{0}\right) \operatorname{Hess}(h)\left(v_{0}\right)\left(v-v_{0}\right)^{t}+R\left(h, \tilde{v}, v_{0}, v\right)
$$

Since $v=\gamma+(n \cdot \gamma) \lambda e$ and $v_{0}=(n \cdot \gamma) \lambda e$,we use properties $\mathbf{P}_{\mathbf{N}} \cdot \mathbf{1}-\mathbf{P}_{\mathbf{N}} \cdot \mathbf{3}$ and (4.5) into (4.16) to obtain

$$
S_{N}^{\epsilon}(2+\delta)=\frac{\lambda^{2}}{2} \frac{1}{N} A_{\epsilon}(2+\delta) \delta \frac{1}{N}+\frac{\lambda^{2}}{2 N} A_{\epsilon}(2+\delta)-A_{\epsilon} \frac{2+\delta}{2 N}\left(\lambda^{2}+\mu^{2}\right)+O\left(n^{2+\delta}\right) .
$$

Taking the limit $\epsilon \rightarrow 0$ we find

$$
S_{N}(2+\delta)=\frac{2+\delta}{2 N}\left[\frac{\delta}{N} \lambda^{2}-\mu^{2}\right] .
$$

Therefore, using Lemma 2.2 we obtain for the limit function $g$ the following inequality

$$
d_{2+\delta}\left(g_{1}(t), g_{2}(t)\right) \leq d_{2+\delta}\left(g_{0_{1}}, g_{0_{2}}\right) \cdot \exp \left\{S_{N}(2+\delta) t\right\}
$$

which can be rewritten in terms of the general Fokker-Planck equation (2.1) for the stationary solution and a general solution as

$$
d_{2+\delta}\left(f(t), B_{\infty}\right) \leq d_{2+\delta}\left(f_{0}, B_{\infty}\right) \cdot \exp \left\{2(2+\delta)\left(\delta \beta^{2}-2 N \alpha^{2}\right) t\right\} .
$$

We are now in a position to prove the main result of this Section, namely the $N$ dimensional version of Theorem 3.2. We can observe that for the linearized equation (2.14) also considered in [15] we obtain the same rate of convergence taking $\delta=0$.

THEOREM 4.2. Let $f(v, t)$ be the solution to equation (2.14), corresponding to the initial datum $f_{0}(v)$ satisfying (1.10), and let us denote by $\bar{B}=D B_{C}^{2-m}$ the stationary solution to the same equation Suppose in addition that

$$
d_{2+\delta}\left(f_{0}, \bar{B}\right)<+\infty, \quad \delta<(2-N+N m) /(1-m) .
$$

Then, the solution $f(v, t)$ converges exponentially to $\bar{B}$ in $d_{2+\delta}-$ metric, and

$$
d_{2+\delta}(f(t), \bar{B}) \leq d_{2+\delta}\left(f_{0}, \bar{B}\right) \exp \left\{\frac{2+\delta}{2}((1-m) \delta-(2-N+N m)) t\right\} .
$$

Moreover, provided $\frac{N}{N+2}<m<1$ the rate of convergence in (4.20) increases in the interval $0<\delta \leq((N+2) m-N) /(2-2 m)$. Likewise, let $f(v, t)$ be the solution to equation (1.6), with initial datum $f_{0}(v)$. Suppose in addition that

$$
d_{2+\delta}\left(f_{0}, B_{C}\right)<+\infty, \quad \delta<(m(N+2)-N) /(1-m) .
$$


Then, the solution $f(v, t)$ converges exponentially to $B_{C}(x)$ in $d_{2+\delta}-m e t r i c$, and

$$
d_{2+\delta}\left(f(t), B_{C}\right) \leq d_{2+\delta}\left(f_{0}, B_{C}\right) \exp \left\{\frac{2+\delta}{2 N}\left(\frac{1-m}{m} \delta-\frac{(2+N) m-N}{m}\right) t\right\} .
$$

Moreover, provided $(N+2) /(N+4)<m<1$ the rate of convergence in (4.22) increases in the interval $0<\delta \leq \frac{(N+4) m-(N+2)}{2-2 m}$.

Proof. The proof of this theorem is an easy generalization of the 1-dimensional case and we can obtain it simply by identifying coefficients, since in section 2.1 we showed that both linear equation $((2.14)$ and (1.6)) can be written, using different scaling, as equation (2.1). For equation (2.14) we have

$$
\alpha^{2}=\frac{\mu^{2}}{2 N^{2}}=\frac{1}{N}-\frac{1-m}{2} \quad \text { and } \quad \beta^{2}=\frac{\lambda^{2}}{2 N^{2}}=\frac{1-m}{2} .
$$

$S_{N}(2+\delta)$ is nonpositive if and only if $\delta \leq \frac{N \mu^{2}}{\lambda^{2}}$. And the minimum value is achieved at $\delta_{0}=\frac{N \mu^{2}}{2 \lambda^{2}}-1$ which is positive if and only if $N \mu^{2}>2 \lambda^{2}$ or equivalently if and only if $N \alpha^{2}>2 \beta^{2}$ (analogous N-dimensional version of (3.41)). And finally, this inequality holds if and only if $m>\frac{N}{N+2}$. On the other hand, since

$$
\mu^{2}=2 N \frac{2-N+N m}{2} \text { and } \quad \lambda^{2}=2 N^{2} \frac{1-m}{2}
$$

we obtain that the rate of convergence $S_{N}(2+\delta)$ takes the form

$$
S_{N}(2+\delta)=\frac{2+\delta}{2}((1-m) \delta-(2-N+N m)) .
$$

The minimum value is achieved at $\delta_{0}=\frac{(2+N) m-N}{2-2 m}$ (see previous computations) and this value is

$$
S_{N}\left(2+\delta_{0}\right)=-\frac{1}{8} \frac{(4-N+(N-2) m)^{2}}{1-m} .
$$

For equation (1.6) we have

$$
\alpha^{2}=\frac{\mu^{2}}{2 N^{2}}=\frac{1}{N}-\frac{1-m}{2 m} \quad \text { and } \quad \beta^{2}=\frac{\lambda^{2}}{2 N^{2}}=\frac{1-m}{2 m}
$$

and in this case $N \alpha^{2}>2 \beta^{2}$ holds if and only if $m>(N+2) /(N+4)$ and the rate of convergence is written as

$$
S_{N}(2+\delta)=\frac{2+\delta}{2 N}\left(\frac{1-m}{m} \delta-\frac{(2+N) m-N}{m}\right) .
$$

The minimum value is now achieved in $\delta_{0}=\frac{(N+4) m-(N+2)}{2-2 m}$ and this value is

$$
S_{N}\left(2+\delta_{0}\right)=-\frac{1}{8} \frac{((2-N)+N m)^{2}}{N m} .
$$


REMARK 4.1. For equation (2.14) the condition $\delta<1$ (which means only equality for the initial mass) is satisfied if $\frac{N-2}{N}<m<\frac{N-1}{N+1}$, while $\delta_{0}<1$ (which means equality of the second moments) requires $\frac{N}{N+2}<m<\frac{2+N}{N+4}$. For equation (1.6) the condition $\delta<1$ holds whenever $\frac{N}{N+2}<m<\frac{N+1}{N+3}$. Likewise $\delta_{0}<1$ if $\frac{N+2}{N+4}<m<\frac{N+4}{N+6}$.

Some comments to Theorem 4.2 are needed. Despite the one-dimensional situation, the boundedness of the Fourier based metric for $s>2$ requires that all the cross moments of both the initial value $f_{0}$ and the stationary state are equal up to $[s]$, where $[s]$ is the entire part of the number $s$. When $s>2$, this condition reads very strong. Thus, the conditions of Theorem 4.2 are equivalent to thats of Theorem 3.2 only for radially symmetric initial data, which, as it can be easily seen looking at the kinetic model, imply that the solution is radially symmetric at any subsequent time. In this case, in fact, only the principal moments of both the initial value and the stationary state need to be equal.

In the general situation, however, it has been shown first for the elastic spatially homogeneous Boltzmann equation for Maxwell molecules [19], and subsequently for the inelastic Boltzmann equation for Maxwell type interactions [4], that the explicitly computable and fast decay to zero of the cross moments can be used to relax the condition of Theorem 4.2 to all the initial data satisfying (1.10). A similar strategy could be applied in the present situation. On the other hand, since we are interested in understanding how the values of the moments improve the rate of convergence to equilibrium, this problem is not of primary importance for our analysis.

Second, as shown in paper [6] for the elastic spatially homogeneous Boltzmann equation for Maxwell molecules, the decay of the Fourier metric, combined with the uniform boundedness of stronger norms, gives rise to precise rates of convergence towards the stationary state in these strong norms. This idea has been recently applied to the spatially uniform Boltzmann equation for inelastic Maxwell model in presence of a thermal bath in [3]. In the purely inelastic setting, however, the lack of an entropy principle, makes it difficult to extend the results of [6] to this case [4]. In the next Section we will give indications on a possible way to obtain decay rates in stronger norm for the target Fokker-Planck equations.

\section{Rate of convergence for general initial values}

\subsection{Propagation of regularity}

The goal of this section is to show that the smoothness of the initial data of the Fokker-Planck equation (2.1) is propagated, so that we have uniform in time bounds on the smoothness. This result, combined with suitable interpolation inequalities [6] can be used to obtain convergence in stronger spaces with almost the same rate of convergence of the metric $d_{2}$. This strategy has been recently applied to the dissipative Boltzmann equation in a thermal bath in [3].

After application of the Fourier transform, the Fokker-Planck equation (2.1) takes the form

$$
\frac{\partial \hat{f}}{\partial t}=-\xi \cdot \nabla_{\xi} \hat{f}-\alpha^{2}|\xi|^{2} \hat{f}+\beta^{2}|\xi|^{2} \Delta_{\xi} \hat{f} .
$$

To simplify computations, thanks to linearity, we can consider separately the real and imaginary parts of $\hat{f}(\xi), \hat{f}(\xi)=a(\xi)+i b(\xi)$. Both the functions $a$ and $b$ then satisfy the same equation (5.1). In the following we argument for $a$ but clearly the same 
holds for $b$. Multiplying the equation (5.1) satisfied by the real part $a(\xi)$ by $2|\xi|^{2 k} a$ one has

$$
\frac{\partial a^{2}}{\partial t}|\xi|^{2 k}=-|\xi|^{2 k} \xi \cdot \nabla_{\xi} a^{2}-2 \alpha^{2}|\xi|^{2 k+2} a^{2}+2 \beta^{2}|\xi|^{2 k+2} a \Delta_{\xi} a
$$

Therefore, integrating over $\mathbb{R}^{N}$ we obtain

$$
\begin{gathered}
\frac{d}{d t} \int_{\mathbb{R}^{N}}|\xi|^{2 k} a^{2} d \xi=-\int_{\mathbb{R}^{N}}|\xi|^{2 k} \xi \cdot \nabla_{\xi} a^{2} d \xi \\
-2 \alpha^{2} \int_{\mathbb{R}^{N}}|\xi|^{2 k+2} a^{2} d \xi+2 \beta^{2} \int_{\mathbb{R}^{N}}|\xi|^{2 k+2} a \Delta_{\xi} a d \xi .
\end{gathered}
$$

Direct computations, integrating by parts produce

$$
\int_{\mathbb{R}^{N}}|\xi|^{2 k} \xi \cdot \nabla_{\xi} a^{2} d \xi=-(N+2 k) \int_{\mathbb{R}^{N}}|\xi|^{2 k} a^{2} d \xi
$$

and

$$
\int_{\mathbb{R}^{N}}|\xi|^{2 k+2} a \Delta_{\xi} a d \xi=(k+1)(N+2 k) \int_{\mathbb{R}^{N}}|\xi|^{2 k} a^{2} d \xi-\int_{\mathbb{R}^{N}}|\xi|^{2 k+2}\left(\nabla_{\xi} a\right)^{2} d \xi .
$$

Hence (5.2) is written as

$$
\begin{gathered}
\frac{d}{d t} \int_{\mathbb{R}^{N}}|\xi|^{2 k} a^{2} d \xi=(N+2 k)\left[1+2 \beta^{2}(k+1)\right] \int_{\mathbb{R}^{N}}|\xi|^{2 k} a^{2} d \xi \\
-2 \alpha^{2} \int_{\mathbb{R}^{N}}|\xi|^{2 k+2} a^{2} d \xi-2 \beta^{2} \int_{\mathbb{R}^{N}}|\xi|^{2 k+2}\left(\nabla_{\xi} a\right)^{2} d \xi .
\end{gathered}
$$

Since $|\hat{f}|^{2}=a^{2}+b^{2}$, we obtain

$$
\begin{array}{r}
\frac{d}{d t} \int_{\mathbb{R}^{N}}|\xi|^{2 k}|\hat{f}|^{2} d \xi=(N+2 k)\left[1+2 \beta^{2}(k+1)\right] \int_{\mathbb{R}^{N}}|\xi|^{2 k}|\hat{f}|^{2} d \xi \\
-2 \alpha^{2} \int_{\mathbb{R}^{N}}|\xi|^{2 k+2}|\hat{f}|^{2} d \xi-2 \beta^{2} \int_{\mathbb{R}^{N}}|\xi|^{2 k+2}\left[\left(\nabla_{\xi} a\right)^{2}+\left(\nabla_{\xi} b\right)^{2}\right] d \xi
\end{array}
$$

The next step is to find suitable lower bounds for the two negative terms in (5.4). We are almost sure that the bounds we will derive in what follows are not new, and one can find them somewhere in the pertinent literature. Due to the simplicity of the proofs, however, we will present them in some detail. We have

LEMma 5.1. Let $f \in L^{1}\left(\mathbb{R}^{N}\right)$ be a probability density function such that, for some $k>0$

$$
\int_{\mathbb{R}^{N}}|\xi|^{2 k+2}\left[\left(\nabla_{\xi} a\right)^{2}+\left(\nabla_{\xi} b\right)^{2}\right] d \xi<\infty
$$

Then, $\int|\xi|^{2 k}|\hat{f}(\xi)|^{2}$ is bounded, and the following inequality holds

$$
\int_{\mathbb{R}^{N}}|\xi|^{2 k}|\hat{f}(\xi)|^{2} d \xi \leq \frac{4}{(N+2 k)^{2}} \int_{\mathbb{R}^{N}}|\xi|^{2 k+2}\left[\left(\nabla_{\xi} a\right)^{2}+\left(\nabla_{\xi} b\right)^{2}\right] d \xi
$$


Proof.

Let $\lambda$ be a positive constant. Then

$$
\begin{aligned}
0 & \leq \int_{\mathbb{R}^{N}}|\xi|^{2 k}\left|\lambda \frac{\xi}{|\xi|} a(\xi)+\right| \xi|\nabla a(\xi)|^{2} d \xi=\lambda^{2} \int_{\mathbb{R}^{N}}|\xi|^{2 k} a^{2}(\xi) d \xi \\
& +\int_{\mathbb{R}^{N}}|\xi|^{2 k+2}|\nabla a(\xi)|^{2} d \xi+2 \lambda \int_{\mathbb{R}^{N}}|\xi|^{2 k} a(\xi) \xi \cdot \nabla a(\xi) d \xi .
\end{aligned}
$$

The last integral into (5.7) can be integrated by parts to give

$$
\begin{gathered}
2 \lambda \int_{\mathbb{R}^{N}}|\xi|^{2 k} a(\xi) \xi \nabla a(\xi) d \xi=\lambda \int_{\mathbb{R}^{N}}|\xi|^{2 k} \xi \cdot \nabla a^{2}(\xi) d \xi= \\
-\lambda(N+2 k) \int_{\mathbb{R}^{N}}|\xi|^{2 k} a^{2}(\xi) d \xi
\end{gathered}
$$

Substituting into (5.7) implies

$$
\int_{\mathbb{R}^{N}}|\xi|^{2 k+2}|\nabla a(\xi)|^{2} d \xi \geq \lambda(N+2 k) \int_{\mathbb{R}^{N}}|\xi|^{2 k} a^{2}(\xi) d \xi-\lambda^{2} \int_{\mathbb{R}^{N}}|\xi|^{2 k} a^{2}(\xi) d \xi
$$

Choosing $\lambda=(N+2 k) / 2$ into (5.8) then proves inequality (5.6).

Let us introduce, for $r \geq 0$, the Sobolev space norms $\|\cdot\|_{H^{r}\left(\mathbb{R}^{N}\right)}$ by

$$
\|f\|_{H^{r}\left(\mathbb{R}^{N}\right)}^{2}=\int_{\mathbb{R}^{N}}|\xi|^{2 r}|\hat{f}(\xi)|^{2} d \xi .
$$

Using inequality (5.6) into inequality (5.4), we can rewrite it as

$$
\frac{d}{d t}\|f\|_{H^{k}\left(\mathbb{R}^{N}\right)}^{2} \leq D\|f\|_{H^{k}\left(\mathbb{R}^{N}\right)}^{2}-2 \alpha^{2}\|f\|_{H^{k+1}\left(\mathbb{R}^{N}\right)}^{2}
$$

where the constant $D$ holds

$$
D=D(N, k, \beta)=(N+2 k)\left(1+2 \beta^{2}(k+1)\right)-\beta^{2} \frac{(N+2 k)^{2}}{2} .
$$

From the differential inequality (5.9) we deduce the uniform boundedness of $\|f\|_{H^{k}\left(\mathbb{R}^{N}\right)}$. The result follows by the following Nash-type inequality.

LEMMA 5.2. Let $f \in L^{1}\left(\mathbb{R}^{N}\right)$ be a probability density function such that, for some $q>0$

$$
\int_{\mathbb{R}^{N}}|\xi|^{q}|\hat{f}(\xi)|^{2} d \xi<\infty
$$

Then, if $0<p<q, \int|\xi|^{p}|\hat{f}(\xi)|^{2}$ is bounded, and the following inequality holds

$$
\int_{\mathbb{R}^{N}}|\xi|^{p}|\hat{f}(\xi)|^{2} d \xi \leq C(p, q, N)\left(\int_{\mathbb{R}^{N}}|\xi|^{q}|\hat{f}(\xi)|^{2} d \xi,\right)^{d(p, q, N)}
$$


where, denoting by $B_{N}$ the measure of the unit ball in $\mathbb{R}^{N}$,

$$
C(p, q, N)=B_{N}^{\frac{q-p}{N+q}}\left(\frac{q-p}{p+N}\right)^{\frac{p+N}{q+N}} \frac{q+N}{q-p},
$$

and

$$
d(p, q, N)=\frac{p+N}{q+N}<1
$$

Proof. Since $f$ is a probability density, $|\hat{f}| \leq 1$. Therefore, for any constant $R>0$ the following bound holds,

$$
\begin{aligned}
\int_{\mathbb{R}^{N}}|\xi|^{p}|\hat{f}(\xi)|^{2} d \xi & \leq R^{p} \int_{|\xi| \leq R}|\hat{f}|^{2} d \xi+R^{p-q} \int_{|\xi|>R}|\xi|^{q}|\hat{f}|^{2} d \xi \\
& \leq R^{p} \int_{|\xi| \leq R} d \xi+R^{p-q} \int_{|\xi|>R}|\xi|^{q}|\hat{f}|^{2} d \xi \\
& \leq R^{N+p}\left|B_{N}\right|+R^{p-q} \int_{\mathbb{R}^{N}}|\xi|^{q}|\hat{f}|^{2} d \xi
\end{aligned}
$$

Optimizing in $R$ we obtain the result.

We can use Lemma 5.2 to obtain a bound in (5.9). To this aim, choosing $p=2 k$ and $q=2 k+2$

$$
\begin{aligned}
\frac{d}{d t}\|f\|_{H^{k}\left(\mathbb{R}^{N}\right)}^{2} & \leq D\|f\|_{H^{k}\left(\mathbb{R}^{N}\right)}^{2}-2 \alpha^{2} C(2 k, 2 k+2, N)^{-1 / d(2 k, 2 k+2, N)}\|f\|_{H^{k}\left(\mathbb{R}^{N}\right)}^{2 / d(2 k, 2 k+2, N)} \\
& \leq D\|f\|_{H^{k}\left(\mathbb{R}^{N}\right)}^{2}\left(1-2 \frac{\alpha^{2}}{D C^{1 / d}}\|f\|_{H^{k}\left(\mathbb{R}^{N}\right)}^{2(1-d) / d}\right)
\end{aligned}
$$

Since $d<1$, the time derivative can growth only if the right-hand side remains positive, that is when

$$
\|f\|_{H^{k}\left(\mathbb{R}^{N}\right)}^{2(1-d) / d} \leq \frac{D C^{1 / d}}{2 \alpha^{2}} .
$$

Clearly this implies a uniform bound on $\|f\|_{H^{k}\left(\mathbb{R}^{N}\right)}^{2}$. We proved

THEOREM 5.3. Let $f_{0}$ be the initial datum for equation (2.1) such that $\left\|f_{0}\right\|_{H^{k}\left(\mathbb{R}^{N}\right)}$ is finite. Then, any probability density $f(t, v)$ solution of $(2.1)$ is bounded in $H^{k}\left(\mathbb{R}^{N}\right)$, and there is a universal constant $D$ so that, for all $t>0$,

$$
\|f(t)\|_{H^{k}\left(\mathbb{R}^{N}\right)} \leq \max \left\{\left\|f_{0}\right\|_{H^{k}\left(\mathbb{R}^{N}\right)}, B\right\},
$$

where

$$
B=\left(\frac{D C^{1 / d}}{2 \alpha^{2}}\right)^{\frac{d}{2(1-d)}} .
$$

$C(2 k, 2 k+2, N)$ and $d(2 k, 2 k+2, N)$ are defined as in Lemma 5.2, while the constant $D$ is defined in (5.10). 


\subsection{Strong convergence to equilibrium}

This section is very short. The regularity result proven in the previous Section can be used together with Theorem 4.2 to obtain rates of convergence to equilibrium in stronger norms. We only need to collect results and to explain how to compute the constants involved in it. The key point are some interpolation inequalities, recently considered in [6] to obtain strong convergence results for the elastic Boltzmann equation for Maxwell molecules. These inequalities have been subsequently been used for obtaining sharp rates of convergence to equilibrium for the dissipative Boltzmann equation in presence of a thermal bath [3]. The first of these inequalities reads

LEMma 5.4. [Control of the $\boldsymbol{H}^{k}$-distance] Let $k, p \geq 0$, then

$$
\|f-g\|_{H^{k}\left(\mathbb{R}^{N}\right)}^{2} \leq C d_{2}(f, g)^{\beta_{1}}\|f-g\|_{H^{k+p}\left(\mathbb{R}^{N}\right)}^{\beta_{2}},
$$

with

$$
\begin{gathered}
\beta_{1}=\frac{4 p}{2 k+2 p+N+4}, \\
\beta_{2}=\frac{2(2 k+N+4)}{2 k+2 p+N+4} \\
C(k, p, N)=\left(\frac{B_{N}}{2 k+N+4}\right)^{\frac{\beta_{1}}{2}}(2 p)^{\frac{\beta_{2}}{2}}+\left(\frac{2 p(2 k+N+4)}{B_{N}}\right)^{\frac{-\beta_{1}}{2}}
\end{gathered}
$$

Proof. For $R>0$ we obtain

$$
\begin{aligned}
\|f-g\|_{H^{k}\left(\mathbb{R}^{N}\right)}^{2} & \leq \int_{|\xi| \leq R}|\xi|^{2 k}|\hat{f}-\hat{g}|^{2} d \xi+\frac{1}{R^{2 p}} \int_{|\xi|>R}|\xi|^{2(k+p)}|\hat{f}-\hat{g}|^{2} d \xi \\
& \leq \int_{|\xi| \leq R}|\xi|^{2(k+2)} d_{2}^{2}(f, g) d \xi+\frac{1}{R^{2 p}}\|f-g\|_{H^{k+p}}^{2}
\end{aligned}
$$

and optimizing on $R$ we obtain the result.

This result shows that the weak $d_{2}$ distance coupled with $\|\cdot\|_{H^{r}}$ smoothness, controls the $H^{k}$ distance for $r$ sufficiently larger than $k$. The next inequality shows that control of sufficiently many moments and control of the $L^{2}$ norm together, control the $L^{1}$ norm.

Lemma 5.5. [Control of the $\boldsymbol{L}^{\mathbf{1}}$-distance [6, Theorem 4.2]] Let $f$ be an integrable function on $\mathbb{R}^{N}$. Then, for all $r>0$

$$
\int_{\mathbb{R}^{N}}|f(v)| d v \leq C(N, r)\left(\int_{\mathbb{R}^{N}}|f(v)|^{2} d v\right)^{2 r /(N+4 r)}\left(\int_{\mathbb{R}^{N}}|v|^{2 r}|f(v)| d v\right)^{N /(N+4 r)}
$$

with

$$
C(N, r)=\left[\left(\frac{N}{4 r}\right)^{4 r /(N+4 r)}+\left(\frac{4 r}{N}\right)^{N /(N+4 r)}\right]\left|B^{N}\right|^{2 r /(N+4 r)} .
$$

Using both Theorems 5.3 and 5.4 with $r=1$, we prove

THEOREM 5.6. Let $f_{0}$ be any initial datum for the Fokker-Planck equation (2.1) satisfying conditions (1.10). Suppose in addition that the conditions of Theorem 4.2 
are satisfied, so that there exists a positive constant $\chi$ such that for some $\delta>0$ there is convergence of the solution $f(v, t)$ in $d_{2+\delta}$-norm towards the steady state $f_{\infty}(v)$ at the exponential rate $\chi$. Let $\epsilon>0$ be given. Then, there is a number $r$ depending only on $\epsilon$ so that whenever

$$
\int_{\mathbb{R}^{N}}|v|^{2} f_{0}(v) d v+\int_{\mathbb{R}^{N}}|k|^{2 r}\left|\hat{f}_{0}(k)\right|^{2} d k<\infty
$$

then it holds that

$$
\left\|f(\cdot, t)-f_{\infty}\right\|_{L^{1}\left(\mathbb{R}^{N}\right)} \leq C_{\epsilon} \exp \{-(1-\epsilon) \chi t\} .
$$

$C_{\epsilon}$ is explicitly computable in terms of the integrals specified above. Moreover, increasing $r$, we obtain the same result if the $L^{1}$-norm is replaced by any $H^{m}$-norm.

Proof. The result follows from the uniform propagation of regularity shown in Theorem 5.3, and from the interpolation inequalities, Lemmas 5.4 and 5.5 above.

\section{Conclusions}

We discussed in this paper the approach to equilibrium for various Fokker-Planck equations with variable coefficient of diffusion. These equations separate in a distinctive way from other Fokker-Planck equations with variable coefficients for the reason that they are obtained by linearizing fast diffusion equations confined by convection. We investigated the approach to equilibrium by using suitable kinetic models of the Boltzmann equation corresponding to nonconservative collision interactions. These interactions are in fact such that the energy is produced by binary collisions. This allows to use for the reckoning of rates of decay Fourier-based metrics which are easy to handle in this kinetic framework. From one side, this seems artificial, since one has to use nonlinear kinetic equations to obtain results on a linear equation. Looking however to previous attempts to the study of this problem, a similar artificial approach based on the nonlinear diffusion equation has been used in [9] to obtain Poincaré inequalities for the linearized fast diffusion equation. As a matter of fact, the analysis of [1] based on the decay of suitable entropies fails for Fokker-Planck equations with polynomial growth of the diffusion coefficient, and requires a further development of entropy techniques.

Acknowledgements: Giuseppe Toscani acknowledges support from the Italian MIUR project "Mathematical Problems of Kinetic Theories". Research of María J. Cáceres is supported by DGI-MEC (Spain) project MTM2005-08024. Various discussions with José Antonio Carrillo, that focused some of the problems treated in this paper and improved it, are kindly acknowledged.

\section{REFERENCES}

[1] A. Arnold, P. Markowich, G. Toscani, A. Unterreiter. On convex Sobolev inequalities and the rate of convergence to equilibrium for Fokker-Planck type equations. Comm. Partial Differential Equations 26 (2001), 43-100.

[2] A. Baldassarri, U. Marini Bettolo Marconi, A. Puglisi, Kinetic models of inelastic gases. Mat. Mod. Meth. Appl. Sci. 12 (2002) 965-983.

[3] M. Bisi, J.A. Carrillo, and G. Toscani, Contractive Metrics for a Boltzmann equation for granular gases: Diffusive equilibria, J. Statist. Phys. 118 (2005), 301-331.

[4] Decay rates in probability metrics towards homogeneous cooling states for the inelastic Maxwell model, J. Statist. Phys. (in press) (2006). 
[5] A. V. Bobylev, The theory of the nonlinear spatially uniform Boltzmann equation for Maxwellian molecules, Sov. Sci. Rev. c 7, (1988) 111-233.

[6] E.A. Carlen, E. Gabetta, G. Toscani, Propagation of smoothness and the rate of exponential convergence to equilibrium for a spatially homogeneous Maxwellian gas, Commun. Math. Phys. 199, (1999) 521-546.

[7] J. A. Carrillo, M. Di Francesco, and G. Toscani, Strict Contractivity of the 2-Wasserstein distance for the porous medium equation by mass-centering, Proc. Amer. math. Soc. (in press) (2005)

[8] J. A. Carrillo, M. P. Gualdani, and G. Toscani, Finite speed of propagation in porous media by mass transportation methods, C. R. Math. Acad. Sci. Paris 338 (2004), no. 10, 815-818.

[9] J. A. Carrillo, C. Lederman, P. Markowich and G. Toscani, Poincaré Inequalities for Linearization of Very Fast Diffusion Equations, Nonlinearity 15 (2002), 565-580.

[10] J. A. Carrillo and G. Toscani, Exponential convergence toward equilibrium for homogeneous Fokker-planck type equations, Math. Meth Appl. Sci. 21 (1998), 1269-1286.

[11] , Asymptotic $L^{1}$-decay of solutions of the porous medium equation to self-similarity, Indiana Univ. Math. J. 49 (2000), no. 1, 113-142.

[12] J.A. Carrillo and J.L. Vázquez, Fine asymptotics for fast diffusion equations, Comm. Partial Differential Equations 28 (2003), 1023-1056.

[13] C. Cercignani, R. Illner, and M. Pulvirenti, The mathematical theory of dilute gases Springer Series in Applied Mathematical Sciences, Vol. 106, Springer-Verlag, (1994).

[14] D. Cordero-Erausquin and R.J McCann, Accelerated diffusion to minimum entropy, personal communication (2005).

[15] J. Denzler and R.J. McCann, Fast diffusion to self-similarity: complete spectrum, long time asymptotics, and numerology, Arch. Rational Mech. Anal. 175 (2005), 301-342.

[16] L. Desvillettes, About the regularizing properties of the non-cut-off Kac equation Comm. Math. Phys., 168(2), (1995) 417-440.

[17] M. Del Pino and J. Dolbeault, Best constants for Gagliardo-Nirenberg inequalities and applications to nonlinear diffusions. J. Math. Pures Appl. (9) 81 (2002), 847-875.

[18] J. Duoandikoetxea and E. Zuazua, Moments, masses de Dirac et décomposition de fonctions, C. R. Acad. Sci. Paris Sér. I Math. 315 (1992), no. 6, 693-698.

[19] E. Gabetta, G. Toscani, B. Wennberg, Metrics for probability distributions and the trend to equilibrium for solutions of the Boltzmann equation, J. Stat. Phys., 81, 901-934, (1995).

[20] T. Goudon, S. Junca, and G. Toscani, Fourier-based distances and Berry-Esseen like inequalities for smooth densities, Monatsh. Math. 135 (2002), no. 2, 115-136.

[21] Y.J. Kim and R.J. McCann, Potential theory and optimal convergence rates in fast nonlinear diffusion, J. Math. Pures Appl. (to appear) (2006)

[22] R.G. Laha, V.K. Rohatgi, Probability Theory, John Wiley and Sons, New York, 1979.

[23] L. Landau, Die kinetische Gleichung für den Fall Coulombscher Wechselwirkung. Phys. Z. Sowjet. 10 (1936), 154. Trad. The transport equation in the case of Coulomb interactions, in D. ter Haar, ed., Collected papers of L.D. Landau, p.163-170. Pergamon Press, Oxford, 1981.

[24] R.J. McCann and D. Slepcev, Nearly optimal convergence rates for centered solutions to the fast-diffusion equations, Preprint, 2005.

[25] L.Pareschi, G. Toscani Self-similarity and power-like tails in nonconservative kinetic models, J. Statist. Phys. (in press) (2006).

[26] A. Pulvirenti, G. Toscani, Asymptotic properties of the inelastic Kac model J. Statist. Phys. 114, (2004) 1453-1480.

[27] G. Toscani, One-dimensional kinetic models with dissipative collisions, M2AN Math. Model. Numer. Anal., 34 (2000), 1277-1292.

[28] G. Toscani, A central limit theorem for solutions fo the porous medium equation, J. Evol. Equ. 5, (2005) 185-203. .

[29] G. Toscani, C. Villani, Probability Metrics and Uniqueness of the Solution to the Boltzmann Equation for a Maxwell Gas, J. Statist. Phys. 94 (1999), 619-637.

[30] J. L. Vázquez, Asymptotic behaviour for the porous medium equation posed in the whole space, J. Evol. Equ. 3, 67-118.

[31] _ Asymptotic behaviour and propagation properties of the one-dimensional flow of gas in a porous medium, Trans. Amer. Math. Soc. 277 (1983), no. 2, 507-527.

[32] C. Villani, Contribution à l'étude mathématique des équations de Boltzmann et de Landau en théorie cinétique des gaz et des plasmas. PhD thesis, Univ. Paris-Dauphine, (1998).

[33] _ On a new class of weak solutions to the spatially homogeneous Boltzmann and Landau equations, Arch. Rational Mech. Anal. 143, (1998) 273-307.

[34] T. P. Witelski and A. J. Bernoff, Self-similar asymptotics for linear and nonlinear diffusion equations, Stud. Appl. Math. 100 (1998), no. 2, 153-193. 